\title{
Electrostatic Analyses for a System Constituted by an Insulating Disk Whose One Plane Surface is Charged and Which is Backed by a Concentric Earthed Metal Disk Smaller Than the Insulating Disk Itself and an Earthed Sphere Approaching the Charged Surface of the Insulating Disk, and Its Application to the Case of a Typical Oxygen Regulator
}

\author{
by \\ Nirmalya Basu \\ Date of Birth: May 3, 1984 \\ Qualification: Master of Engineering (Electronics and Telecommunication Engineering) \\ from Bengal Engineering and Science University, Shibpur \\ Email: nirmalya1basu@gmail.com \\ Mobile: +919007757186 \\ Address: 162/B/185 Lake Gardens, Kolkata - 700045, West Bengal, India.
}

\begin{abstract}
There is a proposition that the possible occurrence of electrostatic discharges inside an oxygen regulator between charged insulating components, such as diaphragms, and earthed metal components, such as the valve stem, could be a potential candidate responsible for oxygen regulator fires. In this paper, the electric field which might be produced at the tip of the valve stem due to charge produced on the diaphragm of a typical oxygen regulator by deposition of charged dust particles or by their impact on the diaphragm or both has been evaluated. The diaphragm, in many cases, is backed partially by a concentric earthed metal disk. The diaphragm has been modelled as an insulating disk. The tip of the valve stem has been modelled as an earthed sphere which is very small compared to the insulating disk and the backing earthed metal disk. An analytical expression for the electric field produced at the point of the sphere nearest to the charged surface of the insulator has been derived. Our expression takes into account not only the effect of the charge on the insulating disk, but also that due to the presence of the backing earthed metal disk. Results for the magnitude of this electric field have been computed for the case of a sample oxygen regulator. An expression for the critical charge density on the surface of the insulating disk has been derived, and its value has been obtained for the case of our sample oxygen regulator. The electric field inside the non-metal diaphragm has also been evaluated. It appears from our analyses that there is a possibility that an electrostatic discharge might occur inside an oxygen regulator, and with an enriched-oxygen atmosphere being present there, such a discharge could also lead to a fire incident.
\end{abstract}

\section{Introduction}

Oxygen regulators, used in places that require oxygen gas of high purity for their operations, such as, welding shops, steel plants and medical facilities, have been responsible for many fire accidents. A number of such incidents have been reported [1 - 5]. In some of these incidents again, the mechanism of ignition could not be firmly established [4, 5]. This indicates that apart from the well-known mechanisms mostly used to explain oxygen regulator fires, namely, 'particle impact' $[1,6]$, 'mechanical impact among regulator components' [6] and 'adiabatic compression' [6], there could be other mechanisms which might also be responsible for such incidents. Two possible mechanisms have been suggested. These are 'flow friction' [6 - 8] and 'tribocharging of particle contaminants' $[9,10]$. 
The last mechanism, i.e., 'tribocharging of particle contaminants' [9, 10], suggests that particle contaminants, while flowing through the oxygen system, might get electrostatically charged due to contact with the inner walls of the system and due to impact amongst themselves. These charged particles could eventually accumulate on the diaphragm of the regulator. This might result in a brush discharge when, during opening of the regulator, the diaphragm comes very close to the valve. The discharge might eventually cause fire due to ignition of dispersed dust particles and of non-metallic parts of the regulator present in the enriched-oxygen atmosphere of the regulator.

An electrostatic discharge inside a regulator is also possible if the insulating diaphragm itself gets charged by the impact of dust particles. That insulating polymer surfaces get charged on being impacted by particles has been experimentally shown using five different polymers (fibreglass, Lucite, Rulon J, Lexan and Teflon) and Mars simulant particles having diameters less than $5 \mu \mathrm{m}$ by making the particles travel at $20 \mathrm{~m} / \mathrm{s}$ in an atmosphere of $\mathrm{CO}_{2}$ at 10 mbar pressure [11].

Whatever the mechanism of charge generation on the diaphragm surface, whether the settling of charged particles on its surface or the charging of the surface itself by impact of particles or both, we have a situation where there is a charge distribution on the surface of an insulator backed partially by an earthed metal plate which charged-insulator-backed-byearthed-metal-plate system could accidentally come close enough to an earthed electrode (which in this case is the valve stem) to cause an electrostatic discharge. Hence, it is important to know the electric field that could be produced in such situations. Since the magnitude of the electric field is maximum at the tip of the electrode, we shall be interested in this magnitude as a discharge will ensue if it exceeds the breakdown strength of the medium. Heidelberg [12] has shown that the magnitude of the electric field at the lowest point of an earthed conducting sphere placed above a uniformly charged circular insulating surface oriented such that the straight line joining its centre with that of the sphere is perpendicular to the charged surface at its centre is given by

$$
E_{V}=\frac{\sigma}{2 \varepsilon}\left\{3-\frac{D}{a}+\frac{(D-a)\left(\frac{D}{a}-3\right)+\frac{R^{2}}{a}}{\sqrt{(D-a)^{2}+R^{2}}}\right\}=\frac{\sigma}{2 \varepsilon} f(a, D, R) \ldots(\mathrm{A}),
$$

where $\sigma$ is the density of charge on the insulating surface, $R$ is the radius of the insulating surface, $a$ is the radius of the earthed sphere, $D$ is the distance between the centre of the sphere and that of the charged surface and $\varepsilon$ is the permittivity of the medium. He has also stated that if the insulating disk is backed by an earthed metal plate of the same radius and if the distance $\eta$ between the charged insulating surface and the metal surface is small compared to the distance between sphere and the insulator, then mathematically, the case may be treated approximately by considering the difference between the effects of two single layers of charge of same magnitude on the sphere, so that the electric field at the lowest point of the earthed sphere is given by 


$$
E_{V}^{\prime}=\frac{\sigma}{2 \varepsilon}[f(a, D, R)-f(a, D+\eta, R)] \ldots(\mathrm{B}),
$$

where the function $f$ has been defined in equation (A).

Oza et al [13] have shown by Computational Fluid Dynamics (CFD) simulation that the concentration of dust particles inside the regulator becomes more or less uniform after its closure. These dust particles gradually settle on the diaphragm surface. Thus, the distribution of charge on the diaphragm surface is more or less uniform.

However, in many cases, the diaphragm is only partially backed by a metal disk as shown in Fig. 1 so that spring-loading is possible.

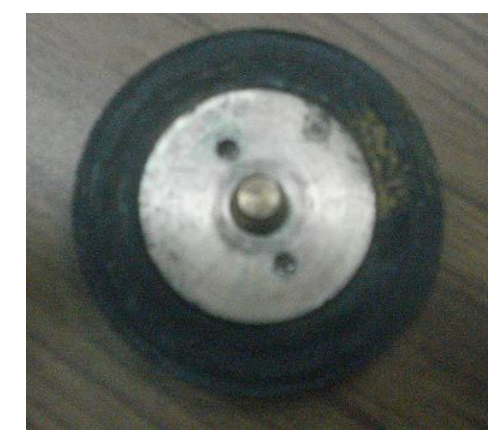

Fig. 1. Photograph of a Regulator Diaphragm Showing the Backing Metal Plate

There is also a metal disk in front of the diaphragm by which the valve stem can be pushed. For ease of analysis, we have ignored its presence as its purpose being only to push the valve stem, its radius can be brought down to that of the valve stem which is very small compared to both the diaphragm and the backing earthed metal disk. Due to the presence of this backing plate - which often gets earthed when a part of an oxygen system - covering some area at the centre of the diaphragm and because of the dust particles being charged, it may be expected that dust particles will settle in greater proportions at and around the centre of the diaphragm than towards its periphery. This is because the electric lines of force resulting from the charged particles will tend to get more concentrated towards the earthed backing plate, thereby causing the particles to be drawn towards it. Hence, the density of charge on the diaphragm surface may be expected to be the maximum at the centre and gradually reducing with increasing distance from it. In this work, we investigate the electric field produced at the tip of the valve stem due to this nature of charge distribution on the diaphragm surface.

Till now, mathematical analysis of the electric field produced due to a charged insulating plate has been done only for cases pertaining to a uniform charge distribution. These results cannot be directly used in our problem as the charge distribution on the insulator (the diaphragm) for our case is non-uniform. We, in this work, mathematically analyse the electric field for a charge distribution which is such that the density of charge is the maximum at the centre of the insulator and gradually diminishing with increasing distance from the centre. Such a charge distribution may be faithfully represented mathematically by the Gaussian function. We model the tip of the valve stem as a very small earthed sphere, the diaphragm as an insulating disk and the earthed backing plate as an 
earthed metal disk smaller than the insulating disk and placed concentrically with the insulating disk.

\section{Statement of the Mathematical Problem}

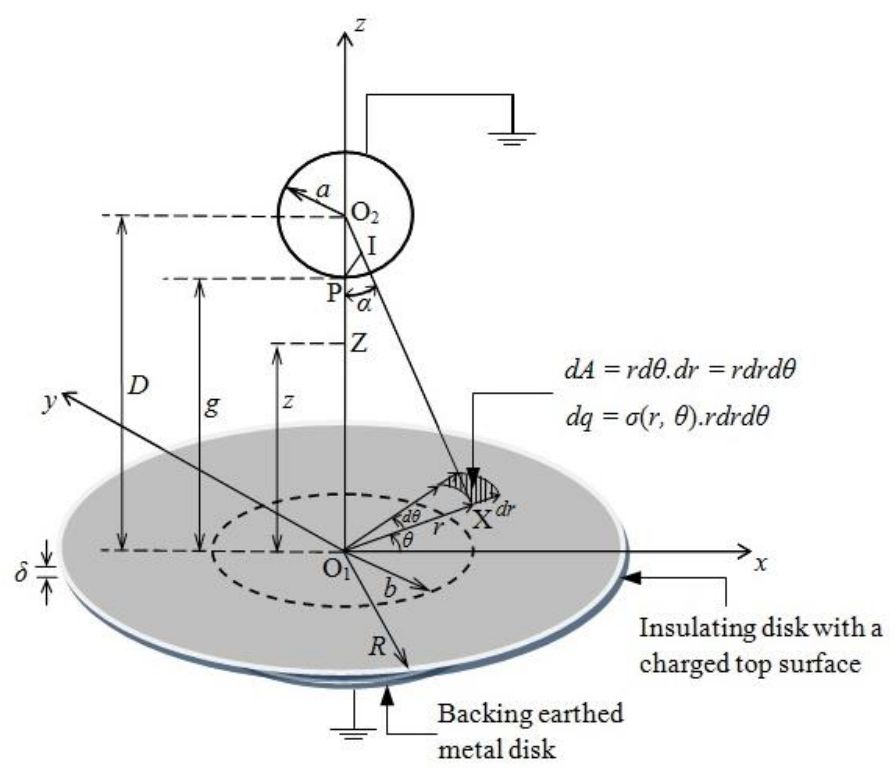

Fig. 2. An Earthed Sphere near a Charged Insulator Partially Backed by an Earthed Metal Disk

We consider a system consisting of an earthed conducting sphere placed near a positively charged insulating disk of thickness $\delta$, which is partially backed by a concentric earthed metal disk, such that the straight line joining the centres of the sphere and the insulator is perpendicular to the insulating surface at its centre as shown in Fig. 2. Let $a$ represent the radius of the sphere, $R$ the radius of the insulating surface, $b(b<R)$ the radius of the backing earthed metal disk, $D$ the distance between the centres of the sphere and the insulator, and $\sigma(r, \theta)$ the surface density of charge on the insulating surface. It is required to find the magnitude of the electric field $E_{\mathrm{P}}$ at the lowest point $\mathrm{P}$ of the sphere for the surface charge density on the insulator given by

$$
\sigma(r, \theta)=\sigma_{0} e^{-r^{2} / 2 s^{2}} \ldots(1)
$$

where $\sigma_{0}$ is the maximum density of charge on the insulator and $s$ is a parameter which controls the width of the "bell" of this bell-shaped charge distribution.

\section{Solution}

First, let us find out the electric field at $\mathrm{P}$ due to the charge on the insulator without considering the effect of the backing earthed metal plate. We shall use the method of images $[14,15]$ for our analysis. The Cartesian coordinate axes are chosen as shown in Fig. 2. Let us consider an element of charge $d q$ at a point $\mathrm{X}$ as shown in Fig. 2. Let $\mathrm{O}_{1} \mathrm{X}=r$, and the angle subtended by $\mathrm{O}_{1} \mathrm{X}$ with the $x$-axis be $\theta$. Then, we have as indicated in Fig. 2 
$d q=\sigma(r, \theta) \cdot r d \theta \cdot d r=\sigma(r, \theta) r d r d \theta$

Let the image charge due to the element of charge $d q$ be located at I. Then, we have $[14,15]$

$O_{2} I=\frac{a^{2}}{O_{2} X}=\frac{a^{2}}{\sqrt{D^{2}+r^{2}}} \cdots(2)$,

and the image charge

$d q^{\prime}=-\frac{a}{\sqrt{D^{2}+r^{2}}} \sigma(r, \theta) r d r d \theta$

Let $\mathrm{Z}$ be any point on the $\mathrm{z}$-axis. Then the potential at $\mathrm{Z}$ due to the element of charge $d q$ and its image $d q^{\prime}$ is given by

$$
d V_{Z, \text { insulator }}=\frac{1}{4 \pi \varepsilon}\left[\frac{\sigma(r, \theta) r d r d \theta}{\sqrt{z^{2}+r^{2}}}-\frac{a \sigma(r, \theta) r d r d \theta}{\sqrt{D^{2}+r^{2}} . I Z}\right] \ldots
$$

Again, we have

$$
\begin{aligned}
I Z^{2} & \left.=O_{2} Z^{2}+O_{2} I^{2}-2 O_{2} Z \cdot O_{2} I \cdot \cos \alpha \text { [where angle } O_{1} O_{2} X=\alpha\right] \\
& =(D-z)^{2}+\frac{a^{4}}{\left(D^{2}+r^{2}\right)}-2 \cdot(D-z) \cdot \frac{a^{2}}{\sqrt{D^{2}+r^{2}}} \cdot \frac{D}{\sqrt{D^{2}+r^{2}}} \\
& =(D-z)^{2}+\frac{a^{4}}{\left(D^{2}+r^{2}\right)}-\frac{2 a^{2} D(D-z)}{\left(D^{2}+r^{2}\right)} \\
& =\frac{(D-z)^{2}\left(D^{2}+r^{2}\right)+a^{4}-2 a^{2} D(D-z)}{D^{2}+r^{2}} \\
\therefore I Z & =\frac{\sqrt{(D-z)^{2}\left(D^{2}+r^{2}\right)+a^{4}-2 a^{2} D(D-z)}}{\sqrt{D^{2}+r^{2}}} \ldots(5)
\end{aligned}
$$

From equations (4) and (5), we have

$$
\begin{gathered}
d V_{Z, \text { insulator }}=\frac{1}{4 \pi \varepsilon}\left[\frac{\sigma(r, \theta) r d r d \theta}{\sqrt{z^{2}+r^{2}}}-\frac{a \sigma(r, \theta) r d r d \theta}{\sqrt{D^{2}+r^{2}}} \cdot \frac{\sqrt{D^{2}+r^{2}}}{\sqrt{(D-z)^{2}\left(D^{2}+r^{2}\right)+a^{4}-2 a^{2} D(D-z)}}\right] \\
=\frac{\sigma(r, \theta) r d r d \theta}{4 \pi \varepsilon}\left[\frac{1}{\sqrt{z^{2}+r^{2}}}-\frac{a}{\sqrt{(D-z)^{2}\left(D^{2}+r^{2}\right)+a^{4}-2 a^{2} D(D-z)}}\right] \ldots \text { (6) }
\end{gathered}
$$

The component of the electric field at $\mathrm{Z}$ along the $\mathrm{z}$-axis is given by

$d E_{Z, \text { insulator }}=-\frac{\partial}{\partial z}\left(d V_{Z, \text { insulator }}\right)$ 
Now, we have

$$
\begin{aligned}
& \frac{\partial}{\partial z}\left(z^{2}+r^{2}\right)^{-1 / 2}=-\frac{1}{2}\left(z^{2}+r^{2}\right)^{-3 / 2} \cdot 2 z=-\frac{z}{\left(z^{2}+r^{2}\right)^{3 / 2}} \ldots(8) \\
& \frac{\partial}{\partial z}\left[\frac{1}{\sqrt{(D-z)^{2}\left(D^{2}+r^{2}\right)+a^{4}-2 a^{2} D(D-z)}}\right] \\
& \quad=-\frac{1}{2\left\{(D-z)^{2}\left(D^{2}+r^{2}\right)+a^{4}-2 a^{2} D(D-z)\right\}^{3 / 2}} \cdot\left[-2(D-z)\left(D^{2}+r^{2}\right)+2 a^{2} D\right] \\
& \quad=-\frac{a^{2} D-(D-z)\left(D^{2}+r^{2}\right)}{\left\{(D-z)^{2}\left(D^{2}+r^{2}\right)+a^{4}-2 a^{2} D(D-z)\right\}^{3 / 2}} \ldots \text { (9) }
\end{aligned}
$$

From equations (6), (7), (8) and (9), we have

$$
\begin{aligned}
& d E_{Z, \text { insulator }}=-\frac{\sigma(r, \theta) r d r d \theta}{4 \pi \varepsilon}\left[-\frac{z}{\left(z^{2}+r^{2}\right)^{3 / 2}}+\frac{a\left\{a^{2} D-(D-z)\left(D^{2}+r^{2}\right)\right\}}{\left\{(D-z)^{2}\left(D^{2}+r^{2}\right)+a^{4}-2 a^{2} D(D-z)\right\}^{3 / 2}}\right] \\
& \text { or, } d E_{Z, \text { insulator }}=\frac{\sigma(r, \theta) r d r d \theta}{4 \pi \varepsilon}\left[\frac{z}{\left(z^{2}+r^{2}\right)^{3 / 2}}-\frac{a\left\{a^{2} D-(D-z)\left(D^{2}+r^{2}\right)\right\}}{\left\{(D-z)^{2}\left(D^{2}+r^{2}\right)+a^{4}-2 a^{2} D(D-z)\right\}^{3 / 2}}\right] \ldots
\end{aligned}
$$

Putting $z=(D-a)$ in equation (10), we have the electric field at the point $\mathrm{P}$ (the lowest point of the earthed sphere) due to the element of charge $d q$ and its image $d q^{\prime}$ as

$$
\begin{aligned}
& d E_{P, \text { insulator }}=\frac{\sigma(r, \theta) r d r d \theta}{4 \pi \varepsilon}\left[\frac{(D-a)}{\left\{(D-a)^{2}+r^{2}\right\}^{3 / 2}}-\frac{a\left\{a^{2} D-a\left(D^{2}+r^{2}\right)\right\}}{\left\{a^{2}\left(D^{2}+r^{2}\right)+a^{4}-2 a^{3} D\right\}^{3 / 2}}\right] \\
& =\frac{\sigma(r, \theta) r d r d \theta}{4 \pi \varepsilon}\left[\frac{(D-a)}{\left\{(D-a)^{2}+r^{2}\right\}^{3 / 2}}-\frac{a^{2}\left\{a D-\left(D^{2}+r^{2}\right)\right\}}{a^{3}\left\{\left(D^{2}+r^{2}\right)+a^{2}-2 a D\right\}^{3 / 2}}\right] \\
& =\frac{\sigma(r, \theta) r d r d \theta}{4 \pi \varepsilon}\left[\frac{(D-a)}{\left\{(D-a)^{2}+r^{2}\right\}^{3 / 2}}-\frac{\left\{a D-\left(D^{2}+r^{2}\right)\right\}}{a\left\{(D-a)^{2}+r^{2}\right\}^{3 / 2}}\right] \\
& =\frac{\sigma(r, \theta) r d r d \theta}{4 \pi \varepsilon\left\{(D-a)^{2}+r^{2}\right\}^{3 / 2}}\left[(D-a)-\frac{\left\{a D-\left(D^{2}+r^{2}\right)\right\}}{a}\right] \\
& =\frac{\sigma(r, \theta) r d r d \theta}{4 \pi \varepsilon\left\{(D-a)^{2}+r^{2}\right\}^{3 / 2}}\left[\frac{\left(a D-a^{2}-a D+D^{2}+r^{2}\right)}{a}\right] \\
& \therefore d E_{P, \text { insulator }}=\frac{\sigma(r, \theta) r d r d \theta}{4 \pi \varepsilon}\left[\frac{\left(D^{2}+r^{2}\right)-a^{2}}{a\left\{(D-a)^{2}+r^{2}\right\}^{3 / 2}}\right] \ldots(11)
\end{aligned}
$$


Hence, the net electric field at point $\mathrm{P}$ due the entire charge on the insulating surface is given by

$$
\begin{aligned}
& E_{P, \text { insulator }}=\int_{0}^{2 \pi R} \int_{0}^{R} \frac{\sigma(r, \theta) r d r d \theta}{4 \pi \varepsilon} \cdot \frac{\left\{\left(D^{2}+r^{2}\right)-a^{2}\right\}}{a\left\{(D-a)^{2}+r^{2}\right\}^{3 / 2}} \\
& \text { or, } E_{P, \text { insulator }}=\frac{1}{4 \pi \varepsilon a} \int_{0}^{2 \pi R} \int_{0}^{R} \sigma(r, \theta) \cdot \frac{r\left\{\left(D^{2}+r^{2}\right)-a^{2}\right\}}{\left\{(D-a)^{2}+r^{2}\right\}^{3 / 2}} \cdot d r d \theta
\end{aligned}
$$

Using the expression for $\sigma(r, \theta)$ given by equation (1) in equation (12), we have

$$
\begin{aligned}
& E_{P, \text { insulator }}=\frac{1}{4 \pi \varepsilon a} \int_{0}^{2 \pi R} \int_{0}^{R} \sigma_{0} e^{-r^{2} / 2 s^{2}} \cdot \frac{r\left\{\left(D^{2}+r^{2}\right)-a^{2}\right\}}{\left\{(D-a)^{2}+r^{2}\right\}^{3 / 2}} \cdot d r d \theta \\
& =\frac{\sigma_{0}}{4 \pi \varepsilon a} \int_{0}^{2 \pi} d \theta \int_{0}^{R} e^{-r^{2} / 2 s^{2}} \cdot \frac{r\left\{\left(D^{2}+r^{2}\right)-a^{2}\right\}}{\left\{(D-a)^{2}+r^{2}\right\}^{3 / 2}} \cdot d r \\
& =\frac{\sigma_{0}}{4 \pi \varepsilon a} \times 2 \pi \times \int_{0}^{R} e^{-r^{2} / 2 s^{2}} \cdot \frac{r\left\{\left(D^{2}+r^{2}\right)-a^{2}\right\}}{\left\{(D-a)^{2}+r^{2}\right\}^{3 / 2}} \cdot d r \\
& =\frac{\sigma_{0}}{2 \varepsilon a} \int_{0}^{R} e^{-r^{2} / 2 s^{2}} \cdot \frac{r\left\{\left(D^{2}+r^{2}\right)-a^{2}\right\}}{\left\{(D-a)^{2}+r^{2}\right\}^{3 / 2}} \cdot d r \\
& =\frac{\sigma_{0}}{2 \varepsilon a} \int_{0}^{R} e^{-r^{2} / 2 s^{2}} \cdot \frac{r\left\{\left(D^{2}-a^{2}\right)+r^{2}\right\}}{\left\{(D-a)^{2}+r^{2}\right\}^{3 / 2}} \cdot d r \\
& =\frac{\sigma_{0}}{2 \varepsilon a} \int_{0}^{R} e^{-r^{2} / 2 s^{2}} \cdot \frac{r\left\{\left(D^{2}-2 D a+a^{2}\right)+r^{2}+2 D a-2 a^{2}\right\}}{\left\{(D-a)^{2}+r^{2}\right\}^{3 / 2}} \cdot d r \\
& =\frac{\sigma_{0}}{2 \varepsilon a} \int_{0}^{R} e^{-r^{2} / 2 s^{2}} \cdot \frac{r\left[\left\{(D-a)^{2}+r^{2}\right\}+2 a(D-a)\right]}{\left\{(D-a)^{2}+r^{2}\right\}^{3 / 2}} \cdot d r \\
& =\frac{\sigma_{0}}{2 \varepsilon a} \int_{0}^{R} e^{-r^{2} / 2 s^{2}} \cdot\left[\frac{1}{\sqrt{(D-a)^{2}+r^{2}}}+\frac{2 a(D-a)}{\left\{(D-a)^{2}+r^{2}\right\}^{3 / 2}}\right] \cdot r d r \\
& =\frac{\sigma_{0}}{2 \varepsilon a}\left[\int_{0}^{R} \frac{e^{-r^{2} / 2 s^{2}} \cdot r d r}{\sqrt{(D-a)^{2}+r^{2}}}+2 a(D-a) \int_{0}^{R} \frac{e^{-r^{2} / 2 s^{2}} \cdot r d r}{\left\{(D-a)^{2}+r^{2}\right\}^{3 / 2}}\right] \\
& =\frac{\sigma_{0}}{2 \varepsilon a}\left[\frac{1}{2} \int_{0}^{R} \frac{e^{-r^{2} / 2 s^{2}} \cdot d\left(r^{2}\right)}{\sqrt{(D-a)^{2}+r^{2}}}+a(D-a) \int_{0}^{R} \frac{e^{-r^{2} / 2 s^{2}} \cdot d\left(r^{2}\right)}{\left\{(D-a)^{2}+r^{2}\right\}^{3 / 2}}\right]
\end{aligned}
$$




$$
\begin{aligned}
& =\frac{\sigma_{0}}{2 \varepsilon a}\left[s^{2} \int_{0}^{R} \frac{e^{-r^{2} / 2 s^{2}} \cdot d\left(r^{2} / 2 s^{2}\right)}{\sqrt{(D-a)^{2}+r^{2}}}+2 a(D-a) s^{2} \int_{0}^{R} \frac{e^{-r^{2} / 2 s^{2}} \cdot d\left(r^{2} / 2 s^{2}\right)}{\left\{(D-a)^{2}+r^{2}\right\}^{3 / 2}}\right] \\
& =\frac{\sigma_{0}}{2 \varepsilon a}\left[s^{2} \int_{0}^{R} \frac{e^{-r^{2} / 2 s^{2}} \cdot d\left(r^{2} / 2 s^{2}\right)}{\sqrt{(D-a)^{2}+2 s^{2}\left(r^{2} / 2 s^{2}\right)}}+2 a(D-a) s^{2} \int_{0}^{R} \frac{e^{-r^{2} / 2 s^{2}} \cdot d\left(r^{2} / 2 s^{2}\right)}{\left\{(D-a)^{2}+2 s^{2}\left(r^{2} / 2 s^{2}\right)\right\}^{3 / 2}}\right] \\
& \left.=\frac{\sigma_{0}}{2 \varepsilon a}\left[s^{2} \int_{0}^{\frac{R^{2}}{2 s^{2}}} \frac{e^{-t} \cdot d t}{\sqrt{(D-a)^{2}+2 s^{2} t}}+2 a(D-a) s^{2} \int_{0}^{\frac{R^{2}}{2 s^{2}}} \frac{e^{-t} \cdot d t}{\left\{(D-a)^{2}+2 s^{2} t\right\}^{3 / 2}}\right] \text { [where } t=\frac{r^{2}}{2 s^{2}}\right] \\
& =\frac{\sigma_{0}}{2 \varepsilon a}\left[\frac{s^{2}}{(D-a)} \int_{0}^{\frac{R^{2}}{2 s^{2}}} \frac{e^{-t} \cdot d t}{\sqrt{1+2\left(\frac{s}{D-a}\right)^{2} t}}+\frac{2 a s^{2}}{(D-a)^{2}} \int_{0}^{\frac{R^{2}}{2 s^{2}}} \frac{e^{-t} \cdot d t}{\left\{1+2\left(\frac{s}{D-a}\right)^{2} t\right\}^{3 / 2}}\right] \\
& \left.=\frac{\sigma_{0}}{2 \varepsilon a}\left[s \beta \int_{0}^{\frac{R^{2}}{2 s^{2}}} \frac{e^{-t} \cdot d t}{\sqrt{1+2 \beta^{2} t}}+2 a \beta^{2} \int_{0}^{\frac{R^{2}}{2 s^{2}}} \frac{e^{-t} \cdot d t}{\left\{1+2 \beta^{2} t\right\}^{3 / 2}}\right] \text { [where } \beta=\frac{s}{D-a}\right] \\
& =\frac{\sigma_{0}}{2 \varepsilon a}\left[\frac{s \beta}{\sqrt{2} \beta} \int_{0}^{\frac{R^{2}}{2 s^{2}}} \frac{e^{-t} \cdot d t}{\sqrt{t+\frac{1}{2 \beta^{2}}}}+\frac{2 a \beta^{2}}{2 \sqrt{2} \beta^{3}} \int_{0}^{\frac{R^{2}}{2 s^{2}}} \frac{e^{-t} \cdot d t}{\left(t+\frac{1}{2 \beta^{2}}\right)^{3 / 2}}\right]
\end{aligned}
$$



$$
\begin{aligned}
& =\frac{\sigma_{0}}{2 \varepsilon a}\left[\frac{s}{\sqrt{2}} \int_{0}^{\frac{R^{2}}{2 s^{2}}}\left(t+\frac{1}{2 \beta^{2}}\right)^{-\frac{1}{2}} e^{-t} \cdot d\left(t+\frac{1}{2 \beta^{2}}\right)+\frac{a}{\sqrt{2} \beta} \int_{0}^{\frac{R^{2}}{2 s^{2}}}\left(t+\frac{1}{2 \beta^{2}}\right)^{-\frac{3}{2}} e^{-t} \cdot d\left(t+\frac{1}{2 \beta^{2}}\right)\right] \\
& =\frac{\sigma_{0}}{2 \varepsilon a}\left[\frac{s}{\sqrt{2}} e^{\frac{1}{2 \beta^{2}}} \int_{0}^{\frac{R^{2}}{2 s^{2}}}\left(t+\frac{1}{2 \beta^{2}}\right)^{-\frac{1}{2}} e^{-\left(t+\frac{1}{2 \beta^{2}}\right)} \cdot d\left(t+\frac{1}{2 \beta^{2}}\right)\right. \\
& \left.+\frac{a}{\sqrt{2} \beta} e^{\frac{1}{2 \beta^{2}}} \int_{0}^{\frac{R^{2}}{2 s^{2}}}\left(t+\frac{1}{2 \beta^{2}}\right)^{-\frac{3}{2}} e^{-\left(t+\frac{1}{2 \beta^{2}}\right)} \cdot d\left(t+\frac{1}{2 \beta^{2}}\right)\right]
\end{aligned}
$$

8 


$$
\begin{aligned}
& =\frac{\sigma_{0}}{2 \varepsilon a}\left[\frac{s}{\sqrt{2}} e^{\frac{1}{2 \beta^{2}}} \int_{\frac{1}{2 \beta^{2}}}^{\left(\frac{R^{2}}{2 s^{2}}+\frac{1}{2 \beta^{2}}\right)} w^{-\frac{1}{2}} e^{-w} d w+\frac{a}{\sqrt{2} \beta} e^{\frac{1}{2 \beta^{2}}} \int_{\frac{1}{2 \beta^{2}}}^{\left(\frac{R^{2}}{2 s^{2}}+\frac{1}{2 \beta^{2}}\right)} w^{-\frac{3}{2}} e^{-w} d w\right]\left[\text { where } w=t+\frac{1}{2 \beta^{2}}\right] \\
& =\frac{\sigma_{0}}{2 \varepsilon a}\left[\frac{s}{\sqrt{2}} e^{\frac{1}{2 \beta^{2}}} \int_{\frac{1}{2 \beta^{2}}}^{\left(\frac{R^{2}}{2 s^{2}}+\frac{1}{2 \beta^{2}}\right)} w^{-\frac{1}{2}} e^{-w} d w\right. \\
& \left.+\frac{a}{\sqrt{2} \beta} e^{\frac{1}{2 \beta^{2}}}\left\{\left.e^{-w} \frac{w^{-\frac{1}{2}}}{\left(-\frac{1}{2}\right)}\right|_{\frac{1}{2 \beta^{2}}} ^{\left(\frac{R^{2}}{2 s^{2}}+\frac{1}{2 \beta^{2}}\right)}-\int_{\frac{1}{2 \beta^{2}}}^{\left(\frac{R^{2}}{2 s^{2}}+\frac{1}{2 \beta^{2}}\right)}\left(-e^{-w}\right) \frac{w^{-\frac{1}{2}}}{\left(-\frac{1}{2}\right)} d w\right\}\right] \\
& =\frac{\sigma_{0}}{2 \varepsilon a}\left[\frac{s}{\sqrt{2}} e^{\frac{1}{2 \beta^{2}}} \int_{\frac{1}{2 \beta^{2}}}^{\left(\frac{R^{2}}{2 s^{2}}+\frac{1}{2 \beta^{2}}\right)} w^{-\frac{1}{2}} e^{-w} d w\right. \\
& +\frac{a}{\sqrt{2} \beta} e^{\frac{1}{2 \beta^{2}}}\left\{-2 e^{-w} w^{-\frac{1}{2}}\left(\begin{array}{l}
\left(\frac{R^{2}}{2 s^{2}}+\frac{1}{2 \beta^{2}}\right) \\
\frac{1}{2 \beta^{2}}
\end{array}-2 \int_{\frac{1}{2 \beta^{2}}}^{\left(\frac{R^{2}}{2 s^{2}}+\frac{1}{2 \beta^{2}}\right)} w^{-\frac{1}{2}} e^{-w} d w\right\}\right] \\
& =\frac{\sigma_{0}}{2 \varepsilon a}\left[\frac{s}{\sqrt{2}} e^{\frac{1}{2 \beta^{2}}} \int_{\frac{1}{2 \beta^{2}}}^{\left(\frac{R^{2}}{2 s^{2}}+\frac{1}{2 \beta^{2}}\right)} w^{-\frac{1}{2}} e^{-w} d w\right. \\
& \left.-\frac{\sqrt{2} a}{\beta} e^{\frac{1}{2 \beta^{2}}}\left\{\frac{e^{-\left(\frac{R^{2}}{2 s^{2}}+\frac{1}{2 \beta^{2}}\right)}}{\sqrt{\frac{R^{2}}{2 s^{2}}+\frac{1}{2 \beta^{2}}}}-\frac{e^{-\frac{1}{2 \beta^{2}}}}{\sqrt{\frac{1}{2 \beta^{2}}}}+\int_{\frac{1}{2 \beta^{2}}}^{\left(\frac{R^{2}}{2 s^{2}}+\frac{1}{2 \beta^{2}}\right)} w^{-\frac{1}{2}} e^{-w} d w\right\}\right] \\
& =\frac{\sigma_{0}}{2 \varepsilon a}\left[\left(\frac{s}{\sqrt{2}}-\frac{\sqrt{2} a}{\beta}\right) e^{\frac{1}{2 \beta^{2}}} \cdot \int_{\frac{1}{2 \beta^{2}}}^{\left(\frac{R^{2}}{2 s^{2}}+\frac{1}{2 \beta^{2}}\right.} w^{-\frac{1}{2}} e^{-w} d w-\frac{\sqrt{2} a}{\beta}\left\{\frac{e^{-\frac{R^{2}}{2 s^{2}}}}{\sqrt{\frac{R^{2}}{2 s^{2}}+\frac{1}{2 \beta^{2}}}}-\sqrt{2} \beta\right\}\right] \\
& =\frac{\sigma_{0}}{2 \varepsilon a}\left[\left(\frac{s}{\sqrt{2}}-\frac{\sqrt{2} a}{\beta}\right) e^{\frac{1}{2 \beta^{2}}}\left\{\Gamma\left(\frac{1}{2}, \frac{1}{2 \beta^{2}}\right)-\Gamma\left(\frac{1}{2}, \frac{R^{2}}{2 s^{2}}+\frac{1}{2 \beta^{2}}\right)\right\}\right. \\
& +\frac{\sqrt{2} a}{\beta}\left\{\sqrt{2} \beta-\frac{e^{-\frac{R^{2}}{2 s^{2}}}}{\sqrt{\frac{R^{2}}{2 s^{2}}+\frac{1}{2 \beta^{2}}}}\right\} \text {, }
\end{aligned}
$$

9 
where $\Gamma(p, x)=\int_{x}^{\infty} t^{p-1} e^{-t} d t$

$$
\begin{aligned}
& =\frac{\sigma_{0}}{2 \varepsilon a}\left[\left\{\frac{s}{\sqrt{2}}-\frac{\sqrt{2} a(D-a)}{s}\right\} e^{\frac{(D-a)^{2}}{2 s^{2}}}\left\{\Gamma\left(\frac{1}{2}, \frac{(D-a)^{2}}{2 s^{2}}\right)-\Gamma\left(\frac{1}{2}, \frac{R^{2}}{2 s^{2}}+\frac{(D-a)^{2}}{2 s^{2}}\right)\right\}\right. \\
& \left.+\frac{\sqrt{2} a(D-a)}{s}\left\{\frac{\sqrt{2} s}{(D-a)}-\frac{e^{-\frac{R^{2}}{2 s^{2}}}}{\sqrt{\frac{R^{2}}{2 s^{2}}+\frac{(D-a)^{2}}{2 s^{2}}}}\right\}\right]
\end{aligned}
$$

[putting the value of $\beta$ ]

$$
\begin{aligned}
& =\frac{\sigma_{0}}{2 \varepsilon a}\left[\left\{\frac{s^{2}-2 a(D-a)}{\sqrt{2} s}\right\} e^{\frac{(D-a)^{2}}{2 s^{2}}}\left\{\Gamma\left(\frac{1}{2}, \frac{(D-a)^{2}}{2 s^{2}}\right)-\Gamma\left(\frac{1}{2}, \frac{(D-a)^{2}+R^{2}}{2 s^{2}}\right)\right\}\right. \\
& \left.+2 a-\frac{\sqrt{2} a(D-a)}{s} \cdot \sqrt{2} s \cdot \frac{e^{-\frac{R^{2}}{2 s^{2}}}}{\sqrt{(D-a)^{2}+R^{2}}}\right] \\
& =\frac{\sigma_{0}}{2 \varepsilon a}\left[\left\{\frac{s^{2}-2 a(D-a)}{\sqrt{2} s}\right\} e^{\frac{(D-a)^{2}}{2 s^{2}}}\left\{\Gamma\left(\frac{1}{2}, \frac{(D-a)^{2}}{2 s^{2}}\right)-\Gamma\left(\frac{1}{2}, \frac{(D-a)^{2}+R^{2}}{2 s^{2}}\right)\right\}\right. \\
& \left.+2 a-\frac{2 a(D-a)}{\sqrt{(D-a)^{2}+R^{2}}} \cdot e^{-\frac{R^{2}}{2 s^{2}}}\right]
\end{aligned}
$$

or, $E_{P, \text { insulator }}=\frac{\sigma_{0}}{2 \varepsilon a}\left[\left\{\frac{s^{2}-2 a(D-a)}{\sqrt{2} s}\right\} e^{\frac{(D-a)^{2}}{2 s^{2}}}\left\{\Gamma\left(\frac{1}{2}, \frac{(D-a)^{2}}{2 s^{2}}\right)-\Gamma\left(\frac{1}{2}, \frac{(D-a)^{2}+R^{2}}{2 s^{2}}\right)\right\}\right.$

$\left.+2 a\left\{1-\frac{(D-a)}{\sqrt{(D-a)^{2}+R^{2}}} \cdot e^{-\frac{R^{2}}{2 s^{2}}}\right\}\right]=\frac{\sigma_{0}}{2 \varepsilon a} \cdot \phi(D, a, R, s)$

... (14), 
where

$$
\begin{aligned}
& \phi(D, a, R, s)=\left[\left\{\frac{s^{2}-2 a(D-a)}{\sqrt{2} s}\right\} e^{\frac{(D-a)^{2}}{2 s^{2}}}\left\{\Gamma\left(\frac{1}{2}, \frac{(D-a)^{2}}{2 s^{2}}\right)-\Gamma\left(\frac{1}{2}, \frac{(D-a)^{2}+R^{2}}{2 s^{2}}\right)\right\}\right. \\
& \left.+2 a\left\{1-\frac{(D-a)}{\sqrt{(D-a)^{2}+R^{2}}} \cdot e^{-\frac{R^{2}}{2 s^{2}}}\right\}\right] \\
& \text { or, } E_{P, \text { insulator }}=\frac{\sigma_{0}}{2 \varepsilon a}\left[\left(\frac{s^{2}-2 a g}{\sqrt{2} s}\right) e^{\frac{g^{2}}{2 s^{2}}}\left\{\Gamma\left(\frac{1}{2}, \frac{g^{2}}{2 s^{2}}\right)-\Gamma\left(\frac{1}{2}, \frac{g^{2}+R^{2}}{2 s^{2}}\right)\right\}\right. \\
& +2 a\left\{1-\frac{g}{\sqrt{g^{2}+R^{2}}} \cdot e^{-\frac{R^{2}}{2 s^{2}}}\right\}=\frac{\sigma_{0}}{2 \varepsilon a} \cdot \psi(g, a, R, s)
\end{aligned}
$$

where $g=D-a$,

and

$$
\psi(g, a, R, s)=\left[\left(\frac{s^{2}-2 a g}{\sqrt{2} s}\right) e^{\frac{g^{2}}{2 s^{2}}}\left\{\Gamma\left(\frac{1}{2}, \frac{g^{2}}{2 s^{2}}\right)-\Gamma\left(\frac{1}{2}, \frac{g^{2}+R^{2}}{2 s^{2}}\right)\right\}+2 a\left\{1-\frac{g}{\sqrt{g^{2}+R^{2}}} \cdot e^{-\frac{R^{2}}{2 s^{2}}}\right\}\right]
$$

Equations (14) and (15) give expressions for the electric field at $\mathrm{P}$, the lowest point of the earthed sphere (as shown in Fig. 2), due only to the charge on the surface of the insulator. Now, we shall proceed to find the net electric field at $\mathrm{P}$ taking into account the effect of the earthed metallic backing disk. No attempt has yet been made to find an exact analytical expression for this net field. We shall find here analytical expressions for the net electric field at $P$.

Finding the Net Electric Field at P Taking into Account the Effect of the Earthed Metallic

\section{Backing Disk}

As has already been mentioned in section 1, Heidelberg [12] has stated that for the case of a charged insulating disk being backed by an earthed metal disk of the same radius, if the distance between the charged insulating surface and the backing metal surface is small compared to the that between sphere and the insulator, then the case may be treated approximately by considering the difference between the effects of two single layers of charge of same magnitude on the sphere, so that the electric field at the lowest point of the earthed sphere is given by equation (B) with the function $f$ appearing in that equation being defined by equation (A). This may be done because the charges on the charged surface of the insulating disk continue to induce charges in the earthed metal disk until the electric field inside the earthed metal disk is zero and the electric field on the surface of the earthed metal disk is perpendicular to this surface, and this state of the electric field is achieved only if a 
charge of a surface density which is same in magnitude as, but opposite in sign to that of the charge on the charged surface of the insulating disk is induced on the surface of the earthed metal disk facing the charged surface of the insulating disk and the charge induced on the other bounding surfaces of the earthed metal disk is zero. (This is so only if the distance between the surface of charge on the charged surface of the insulating disk and the surface of the earthed metal disk facing this surface of charge is very small compared to the radii of these two surfaces.) The charge induced on the surface of the backing earthed metal facing the charged surface of the insulating disk is too tightly bound to the charge on the insulating disk to be able to move freely in the metal, and hence, it may be treated as though it were residing on an insulating surface. Using this approach and considering the charge induced on the earthed backing disk to be almost solely due to the charge residing on the insulator immediately above the metal disk (and considering the effect of the charge on the insulator residing beyond the distance $b$ from the centre to be negligible so far as the production of the induced charge is concerned), an assumption which might be considered valid to a good approximation for $\delta \ll D, R, b$, which is true for our case, the net electric field at $\mathrm{P}$ is given by

$$
\begin{aligned}
& E_{P, \text { net }}=\frac{\sigma_{0}}{2 \varepsilon a}[\phi(D, a, R, s)-\phi(D+\delta, a, b, s)] \ldots(16) \\
& o r, E_{P, \text { net }}=\frac{\sigma_{0}}{2 \varepsilon a}[\psi(g, a, R, s)-\psi(g+\delta, a, b, s)] \ldots
\end{aligned}
$$

If we wish to consider the image charges produced in the earthed metal disk instead of the charges induced on its upper surface - the surface facing the charges on the insulating disk to calculate the electric field, we will have the following expression for the net electric field at P:

$$
\begin{aligned}
& E_{P, \text { net }}=\frac{\sigma_{0}}{2 \varepsilon a}[\phi(D, a, R, s)-\phi(D+2 \delta, a, b, s)] \ldots(18) \\
& \text { or, } E_{P, \text { net }}=\frac{\sigma_{0}}{2 \varepsilon a}[\psi(g, a, R, s)-\psi(g+2 \delta, a, b, s)] \ldots
\end{aligned}
$$

This is because each element of charge (having a charge $\sigma(r, \theta) \cdot r d r d \theta$ ) on the charged surface of the insulating disk upto the distance $b$ from the centre produces an image charge which has a charge of a magnitude same as, and a sign opposite to that of the element of charge itself on the side of the earthed metal disk surface facing the charged surface of the insulating disk opposite to that of the charged surface of the insulating disk at a distance $\delta$ from this earthed metal disk surface on the straight line which passes through the said element of charge and is perpendicular to this surface of the earthed metal disk $[14,15]$. We note that for the elements of charge on the insulating disk (each having a charge $\sigma(r, \theta) . r d r d \theta$ ) that lie towards the periphery of the earthed metal disk, the surface of the earthed metal disk facing these charges cannot be considered to be really forming a closed surface which bound spaces internally and externally, which is a condition which is necessary to ensure that the electric field produced 
on this metal surface due to each of these elements of charge is the same as that obtained on this surface by adding the electric fields due to this element of charge and another charge which we call its image which has a magnitude and a sign of charge and a position such that the surface described by this surface of the earthed metal disk (the surface of the earthed metal disk facing the charged surface of the insulating disk) is an equipotential surface and has a potential same as that of the metal disk (which in this case is zero). This requirement in using the method of images is demanded by the following theorem:

"If the value of the potential $V$ is known at every point on a number of closed surfaces by which a space is bounded internally and externally, there is only one value for $V$ at every point of this intervening space, which satisfies the condition that $\nabla^{2} V$ either vanishes or has an assigned value at every point of this space." [15]

Thus, in using the method of images for the elements of charge on the charged surface of the insulating disk that lie towards the periphery of the earthed metal disk, we have made the assumption that the surface of the earthed metal disk facing these elements of charge to be forming a closed surface which bound spaces internally and externally despite these elements of charge being near to the boundary of this surface. This assumption is valid as each of these elements of charge has infinitesimal charge and, hence, has a negligible effect on the points in the space on the side of the earthed metal disk opposite to that in which each of these elements of charge is located. These images, again, give rise to the distribution profile of the induced charge in the earthed metal disk as has been described above- each point on the surface of the earthed metal disk facing the charged surface of the insulating disk having a surface charge density having magnitude same as and sign opposite to that at the point on the charged surface of the insulating disk right above it and the other surfaces of the earthed metal disk bounding it having zero charge.

We assume the effect of the charge on the insulator beyond the distance $b$ from the centre in producing image charges in the earthed backing disk to be negligible. The assumption is valid to a good approximation in our case since we have here $\delta<<R, b$. That it is true may be understood by reasoning that in such a case, the charges on the surface of the insulator right above the earthed metal disk will have the maximum effect in producing the induced charges on the surface of the earthed metal disk and the charges on the surface of the insulator beyond the distance $b$ from the centre will have little effect in producing these induced charges, and that image charges have the effect of only producing this induced charge, and hence, it must be so that image charges are produced in the earthed metal disk almost solely due to the charges on the surface of the insulator right above the earthed metal disk, and very little due to the charges on the surface of the insulator beyond the distance $b$ from the centre. Our approximation - that of neglecting the effect of the charges on the surface of the insulator beyond distance $b$ from the centre in producing the image charges in the earthed metal disk - can only have the effect of varying, if at all, only slightly the electric field and the induced-charge distribution profile on the surface of the earthed metal disk, but these variations can cause very little changes to the electric field at $\mathrm{P}$ - the question that we are interested in here. 
In the above analysis, the effect of reflection of the image charges produced in the earthed sphere on the earthed backing disk and the reflection of these new images back on the sphere, and such reflection of image charges going on on the two earthed metal surfaces ad infinitum has been neglected. Now, we shall try to take care of this effect. We do our analysis below.

Our system may be reproduced as follows:

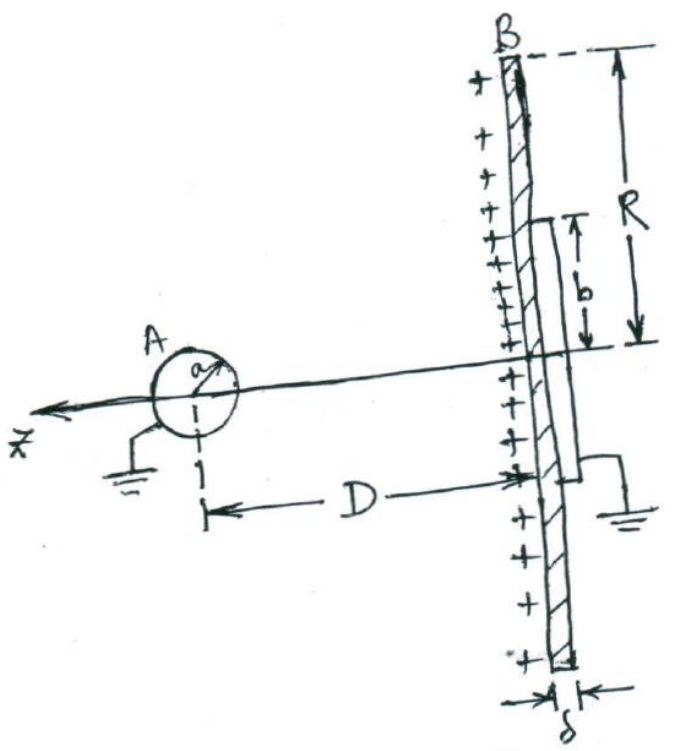

$$
\begin{aligned}
& a \ll D, b, R \\
& \delta \ll D, b, R .
\end{aligned}
$$

Fig. 3. An Earthed Sphere in Front of a Charged Insulating Disk Partially Backed by an Earthed Metal Disk (Figure Depicting the Longitudinal Section of the System Shown in Fig. 2)

As has been shown in Fig. 3, we assume that $a, \delta<<D, b, R$. The assumption that $a, \delta<<D$ is valid for our system of interest for all cases except when the sphere is very close to the insulating disk. The other assumptions are always valid for our system. We draw the following figure (Fig. 4) for ease of visualization in doing our analysis. 


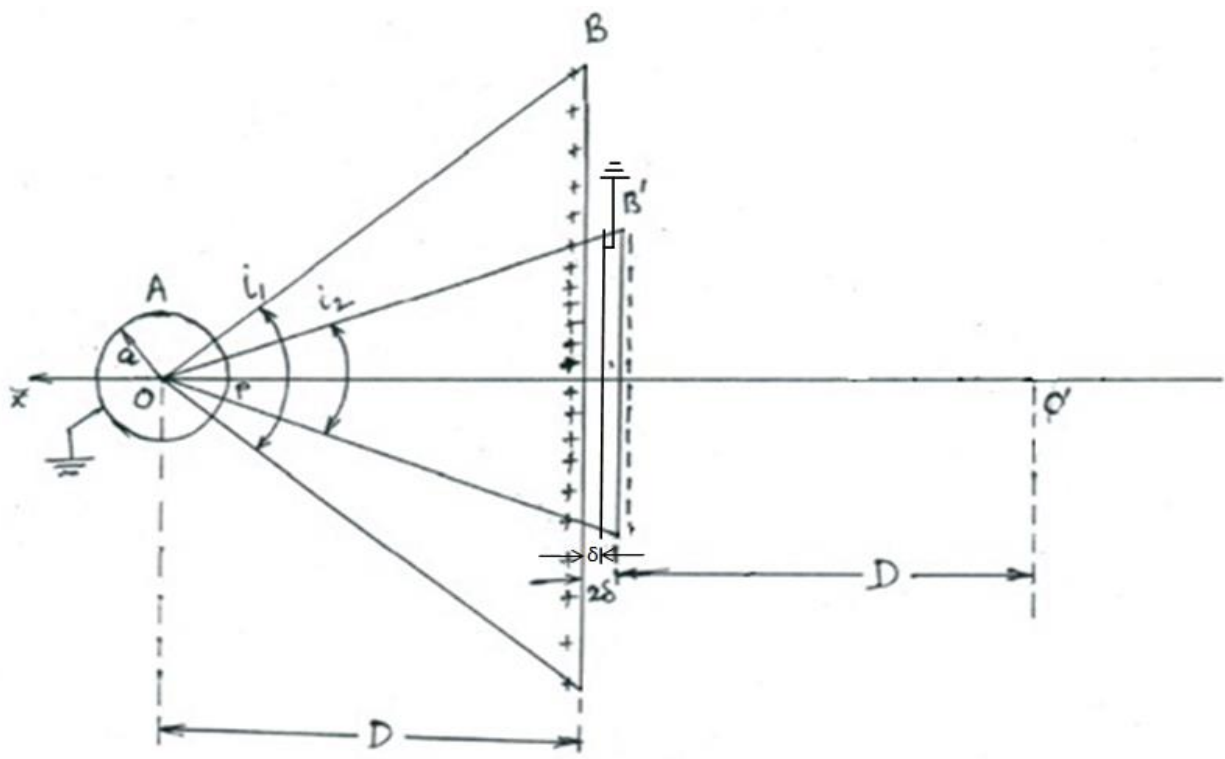

Fig. 4. Figure Meant for Ease of Visualization in Using the Method of Images such that the Effect of Reflection of the Image Charges Produced in the Earthed Metal Sphere on the Earthed Metal Backing Disk and the Reflection of These New Image Charges Back on the Sphere, and Such Reflection of Image Charges Going On on The Two Earthed Metal Surfaces Ad Infinitum Is Taken Care Of (A Denoting the Earthed Metal Sphere in the System of Our Interest, B Denoting the Surface of Charge on the Insulating Disk, B' Denoting the Surface of Image Charges Produced Due To Reflection of The Charges on the Surface of Charge B Upto the Distance $b$ From the Centre on the Earthed Metal Disk, $i_{1}$ and $i_{2}$ Denoting the Angles Subtended by the Surfaces B and B' Respectively at Centre O of the Earthed Metal Sphere A)

As has been explained above, $\mathrm{B}^{\prime}$ will have the same charge distribution profile (in magnitude) as $\mathrm{B}$ has up to the distance $b$ from the centre, but the charges on $\mathrm{B}^{\prime}$ will differ from those on B in sign.

The electric field at point $\mathrm{P}$ will be produced by the charge surfaces $\mathrm{B}$ and $\mathrm{B}^{\prime}$, the image charges $q_{i_{1}}$ and $q_{i_{2}}$ produced by these in sphere A, the images produced in the earthed metal disk by these image charges, the images produced by these back in sphere A, their images produced again in the earthed metal disk, and so going on ad infinitum. The electric field at $\mathrm{P}$ is, thus, given by

$E_{P, \text { net }}=E_{B, q_{i_{1}}}+E_{B^{\prime}, q_{i_{2}}}+E_{I_{A}-\left\{Q_{1}\right\}}+E_{I_{o^{\prime}}} \ldots(20)$

where

$E_{B, q_{i}}$ is the electric field produced at $\mathrm{P}$ by the charge surface B and its image charge $q_{i_{1}}$ in sphere A,

$E_{B^{\prime}, q_{i_{2}}}$ is the electric field produced at $\mathrm{P}$ by the charge surface $\mathrm{B}^{\prime}$ and its image charge $q_{i_{2}}$ in sphere A,

$E_{I_{A}-\left\{Q_{1}\right\}}$ is the electric field produced at $\mathrm{P}$ by all image charges in sphere $\mathrm{A}$ except $Q_{1}\left(=q_{i_{1}}+q_{i_{2}}\right)$, and 
$E_{I_{o}}$ is the electric field produced at $\mathrm{P}$ by all image charges at point $\mathrm{O}^{\prime}$.

We have

$$
\begin{aligned}
& E_{B, q_{i_{1}}}=\frac{\sigma_{0}}{2 \varepsilon a}\left[\frac{\left\{s^{2}-2 a(D-a)\right\}}{\sqrt{2} s} \cdot e^{\frac{(D-a)^{2}}{2 s^{2}}}\left\{\Gamma\left(\frac{1}{2}, \frac{(D-a)^{2}}{2 s^{2}}\right)-\Gamma\left(\frac{1}{2}, \frac{(D-a)^{2}+R^{2}}{2 s^{2}}\right)\right\}\right. \\
& \left.+2 a\left\{1-\frac{(D-a)}{\sqrt{(D-a)^{2}+R^{2}}} \cdot e^{-\frac{R^{2}}{2 s^{2}}}\right\}\right]=\frac{\sigma_{0}}{2 \varepsilon a} \cdot \phi(D, a, R, s)
\end{aligned}
$$

[using equation (14)]

$$
\begin{aligned}
& E_{B^{\prime}, q_{i 2}}=-\frac{\sigma_{0}}{2 \varepsilon a}\left[\frac{\left\{s^{2}-2 a(\overline{D+2 \delta}-a)\right\}}{\sqrt{2} s} \cdot e^{\frac{(\overline{D+2 \delta}-a)^{2}}{2 s^{2}}}\left\{\Gamma\left(\frac{1}{2}, \frac{(\overline{D+2 \delta}-a)^{2}}{2 s^{2}}\right)-\Gamma\left(\frac{1}{2}, \frac{(\overline{D+2 \delta}-a)^{2}+b^{2}}{2 s^{2}}\right)\right\}\right. \\
& +2 a\left\{1-\frac{(\overline{D+2 \delta}-a)}{\sqrt{(\overline{D+2 \delta}-a)^{2}+b^{2}}} \cdot e^{-\frac{b^{2}}{2 s^{2}}}\right\}=-\frac{\sigma_{0}}{2 \varepsilon a} \cdot \phi(\overline{D+2 \delta}, a, b, s)
\end{aligned}
$$

... (22) [using equation (14)]

We have, thus, found expressions for the first two terms for $E_{P, \text { net }}$ given in equation (20). Now, we shall proceed to find out expressions for the remaining two terms given in this equation, namely, $E_{I_{A}-\left\{Q_{1}\right\}}$ and $E_{I_{o^{\prime}}}$. In order that we may do so, we start by finding out expressions for $q_{i_{1}}$ and $q_{i_{2}}$.

We first proceed to find out $q_{i_{1}}$, i.e., the image charge produced by the charge surface $B$ in sphere A. Using equation (3), we have

$$
d q_{i_{1}}=-\frac{a}{\sqrt{D^{2}+r^{2}}} \sigma(r, \theta) r d r d \theta
$$

This image charge is located on the line segment joining the centre $\mathrm{O}$ of sphere $\mathrm{A}$ and the element of charge $d q$ on the surface $\mathrm{B}$ at a distance $\frac{a^{2}}{\sqrt{D^{2}+r^{2}}}$ from O. Since we have assumed that $a \ll D, R, b$, and also that $a$ itself is very small, this image charge may be assumed to be located almost at the centre $\mathrm{O}$ of the sphere. The total image charge produced in A by the charge surface $B$ is given by

$$
q_{i_{1}}=-\int_{0}^{2 \pi} \int_{0}^{R} \frac{a}{\sqrt{D^{2}+r^{2}}} \sigma(r, \theta) r d r d \theta \ldots
$$




$$
\begin{aligned}
& o r, q_{i_{1}}=-\int_{0}^{2 \pi R} \int_{0}^{2} \frac{a}{\sqrt{D^{2}+r^{2}}} \cdot \sigma_{0} e^{-\frac{r^{2}}{2 s^{2}}} \cdot r d r d \theta \\
& =-a \sigma_{0} \int_{0}^{2 \pi} d \theta \int_{0}^{R} \frac{e^{-\frac{r^{2}}{2 s^{2}}} \cdot r d r}{\sqrt{D^{2}+r^{2}}} \\
& =-a \sigma_{0}(2 \pi) \int_{0}^{R} \frac{e^{-\frac{r^{2}}{2 s^{2}}} \cdot r d r}{\sqrt{D^{2}+r^{2}}} \\
& =-a \sigma_{0} \pi \int_{0}^{R} \frac{e^{-\frac{r^{2}}{2 s^{2}}} \cdot 2 r d r}{\sqrt{D^{2}+r^{2}}} \\
& =-a \sigma_{0} \pi \int_{0}^{R} \frac{e^{-\frac{r^{2}}{2 s^{2}}} \cdot d\left(r^{2}\right)}{\sqrt{D^{2}+r^{2}}} \\
& =-2 s^{2} a \sigma_{0} \pi \int_{0}^{R} \frac{e^{-\frac{r^{2}}{2 s^{2}}} \cdot d\left(\frac{r^{2}}{2 s^{2}}\right)}{\sqrt{D^{2}+2 s^{2}\left(\frac{r^{2}}{2 s^{2}}\right)}} \\
& =-2 a s^{2} \sigma_{0} \pi \int_{0}^{\frac{R^{2}}{2 s^{2}}} \frac{e^{-t} \cdot d t}{\sqrt{D^{2}+2 s^{2} t}} \text { [where } t=\frac{r^{2}}{2 s^{2}} \text { ] } \\
& =-\sqrt{2} a s \sigma_{0} \pi \int_{0}^{\frac{R^{2}}{22^{2}}} \frac{e^{-t} \cdot d t}{\sqrt{t+\frac{D^{2}}{2 s^{2}}}} \\
& =-\sqrt{2} a s \sigma_{0} \pi \int_{0}^{\frac{R^{2}}{2 s^{2}}}\left(t+\frac{D^{2}}{2 s^{2}}\right)^{-\frac{1}{2}} \cdot e^{-t} \cdot d\left(t+\frac{D^{2}}{2 s^{2}}\right) \\
& =-\sqrt{2} a s \sigma_{0} \pi e^{\frac{D^{2} s^{2}}{2 s^{2}}} \int_{0}^{\frac{R^{2}}{2 x^{2}}}\left(t+\frac{D^{2}}{2 s^{2}}\right)^{-\frac{1}{2}} \cdot e^{-\left(t+\frac{D^{2}}{2 s^{2}}\right)} \cdot d\left(t+\frac{D^{2}}{2 s^{2}}\right) \\
& =-\sqrt{2} a s \sigma_{0} \pi e^{\frac{D^{2}}{2 s^{2}}} \int_{\frac{D^{2}}{2 s^{2}}}^{\frac{\left(R^{2}+D^{2}\right)}{2 \cdot 2}} w^{-\frac{1}{2}} \cdot e^{-w} \cdot d w\left[\text { where } w=t+\frac{D^{2}}{2 s^{2}}\right] \\
& o r, q_{i_{1}}=-\sqrt{2} a s \sigma_{0} \pi e^{\frac{D^{2}}{2 s^{2}}}\left\{\Gamma\left(\frac{1}{2}, \frac{D^{2}}{2 s^{2}}\right)-\Gamma\left(\frac{1}{2}, \frac{R^{2}+D^{2}}{2 s^{2}}\right)\right\} \ldots
\end{aligned}
$$


Similarly, it can be shown that the image charge produced in sphere A by the surface of charge $\mathrm{B}^{\prime}$ is given by

$q_{i_{2}}=\sqrt{2} a s \sigma_{0} \pi e^{\frac{(D+2 \delta)^{2}}{2 s^{2}}}\left\{\Gamma\left(\frac{1}{2}, \frac{(D+2 \delta)^{2}}{2 s^{2}}\right)-\Gamma\left(\frac{1}{2}, \frac{b^{2}+(D+2 \delta)^{2}}{2 s^{2}}\right)\right\} \ldots$

and that this charge too may be assumed to be located almost at the centre $\mathrm{O}$ of this sphere.

We, thus, have a charge $Q_{1}\left(=q_{i_{1}}+q_{i_{2}}\right)$ located almost at the centre $\mathrm{O}$ of the earthed metal sphere A. $\mathrm{O}$ is located at a distance $(D+\delta)$ from the earthed metal disk. The charge $Q_{1}$ will reflect on the surface of the earthed metal disk, and will, thus, produce an image charge of value $\left(-Q_{1}\right)$ at point $\mathrm{O}^{\prime}$ which is located at a distance $(D+\delta)$ from the earthed metal disk on the side of it opposite to that where sphere $\mathrm{A}$, and hence, point $\mathrm{O}$ and charge $Q_{1}$ are located. (Kindly refer to fig. 4.) This image charge of value $\left(-Q_{1}\right)$ produced at $\mathrm{O}^{\prime}$ will reflect on the surface of the earthed sphere A, and will, thus, produce in it an image charge of value $\frac{a}{2(D+\delta)} Q_{1}$ at a point located at a distance $\frac{a^{2}}{2(D+\delta)}$ from $\mathrm{O}$ on the line joining $\mathrm{O}$ and $\mathrm{O}^{\prime}$. Since we have assumed for our system $a \ll D, R, b$, and also that $a$ itself is very small, the distance of this image charge from $\mathrm{O}$ may be considered negligible, and this image charge may be assumed to be located almost at $\mathrm{O}$. This image charge of value $\frac{a}{2(D+\delta)} Q_{1}$ located at $\mathrm{O}$ will reflect on the earthed metal disk, and will, thus, produce at $\mathrm{O}^{\prime}$ an image charge of value $\left\{-\frac{a}{2(D+\delta)} Q_{1}\right\}$. Reflections of image charges will continue to occur on the earthed metal sphere A and on the earthed metal disk following the manner described here ad infinitum. Since we have assumed for our system that $a<<, R, b$, and also that $a$ itself is very small, we consider for all image charges in the earthed sphere A their distances from the sphere's centre $O$ to be negligible. In other words, we assume in our calculations that in the earthed sphere A, all the images are produced almost at its centre O. This also results in underestimation of the magnitudes of the image charges by a certain extent. However, because of our assumption that $a<<D, R, b$, we may consider this underestimation to be negligible. Based on this assumption, we have an infinite number of image charges produced at points $\mathrm{O}$ and $\mathrm{O}^{\prime}$ as shown below in Table $\mathrm{I}$. 
Table I: Image Charges Produced at Points $O$ and $O^{\prime}$

\begin{tabular}{|c|c|}
\hline Image Charges Produced at Point $O$ & Image Charges Produced at Point $O^{\prime}$ \\
\hline$Q_{1}$ & $\begin{array}{l}-Q_{1} \\
\text { [image produced due to reflection of } Q_{1} \text { on the } \\
\text { earthed metal disk] }\end{array}$ \\
\hline $\begin{array}{c}\frac{a}{2(D+\delta)} Q_{1} \\
\text { [image produced due to reflection of }\left(-Q_{1}\right) \text { on the } \\
\text { earthed metal sphere } \mathrm{A}]\end{array}$ & $\begin{array}{l}\qquad-\frac{a}{2(D+\delta)} Q_{1} \\
\text { [image produced due to reflection of } \\
\frac{a}{2(D+\delta)} Q_{1} \\
\text { on the earthed metal disk] }\end{array}$ \\
\hline $\begin{array}{c}\frac{a^{2}}{\{2(D+\delta)\}^{2}} Q_{1} \\
\text { [image produced due to reflection of } \\
\left\{-\frac{a}{2(D+\delta)} Q_{1}\right\} \\
\text { on the earthed metal sphere } \mathrm{A}]\end{array}$ & $\begin{array}{c}\qquad-\frac{a^{2}}{\{2(D+\delta)\}^{2}} Q_{1} \\
\text { [image produced due to reflection of } \\
\frac{a^{2}}{\{2(D+\delta)\}^{2}} Q_{1} \\
\text { on the earthed metal disk] }\end{array}$ \\
\hline $\begin{array}{c}\frac{a^{3}}{\{2(D+\delta)\}^{3}} Q_{1} \\
\text { [image produced due to reflection of } \\
\left\{-\frac{a^{2}}{\{2(D+\delta)\}^{2}} Q_{1}\right\} \\
\text { on the earthed metal sphere A] }\end{array}$ & $\begin{array}{c}-\frac{a^{3}}{\{2(D+\delta)\}^{3}} Q_{1} \\
\text { [image produced due to reflection of } \\
\frac{a^{3}}{\{2(D+\delta)\}^{3}} Q_{1} \\
\text { on the earthed metal disk] }\end{array}$ \\
\hline $\begin{array}{c}\frac{a^{4}}{\{2(D+\delta)\}^{4}} Q_{1} \\
\text { [image produced due to reflection of } \\
\left\{-\frac{a^{3}}{\{2(D+\delta)\}^{3}} Q_{1}\right\} \\
\text { on the earthed metal sphere } \mathrm{A}]\end{array}$ & $\begin{array}{l}\qquad-\frac{a^{4}}{\{2(D+\delta)\}^{4}} Q_{1} \\
\text { [image produced due to reflection of } \\
\frac{a^{4}}{\{2(D+\delta)\}^{4}} Q_{1} \\
\text { on the earthed metal disk] }\end{array}$ \\
\hline $\begin{array}{l}\cdot \\
\cdot\end{array}$ & $\begin{array}{l}\cdot \\
\cdot\end{array}$ \\
\hline
\end{tabular}

We then have

$$
\begin{aligned}
& E_{I_{A}-\left\{Q_{1}\right\}}=\frac{1}{4 \pi \varepsilon a^{2}}[ \frac{a}{2(D+\delta)} \cdot Q_{1}+\frac{a^{2}}{\{2(D+\delta)\}^{2}} \cdot Q_{1}+\frac{a^{3}}{\{2(D+\delta)\}^{3}} \cdot Q_{1} \\
&\left.+\frac{a^{4}}{\{2(D+\delta)\}^{4}} \cdot Q_{1}+\cdots \text { to } \infty \text { terms }\right] \\
& \text { or, } E_{I_{A}-\left\{Q_{1}\right\}}=\frac{Q_{1}}{4 \pi \varepsilon a^{2}}\left[\frac{a}{2(D+\delta)}+\frac{a^{2}}{\{2(D+\delta)\}^{2}}+\frac{a^{3}}{\{2(D+\delta)\}^{3}}+\frac{a^{4}}{\{2(D+\delta)\}^{4}}+\cdots \text { to } \infty \text { terms }\right]
\end{aligned}
$$




$$
\begin{aligned}
& =\frac{Q_{1}}{4 \pi \varepsilon a^{2}} \cdot \frac{\frac{a}{2(D+\delta)}}{\left[1-\frac{a}{2(D+\delta)}\right]} \\
& =\frac{Q_{1}}{4 \pi \varepsilon a^{2}} \cdot \frac{\frac{a}{2(D+\delta)}}{\frac{2(D+\delta)-a\}}{2(D+\delta)}} \\
& =\frac{Q_{1}}{4 \pi \varepsilon a^{2}} \cdot \frac{a}{\{2(D+\delta)-a\}} \\
\text { or, } E_{I_{A}-\left\{Q_{1}\right\}} & =\frac{1}{4 \pi \varepsilon a^{2}} \cdot\left[\frac{a}{\{2(D+\delta)-a\}} \cdot Q_{1}\right] \ldots(29) \\
\text { or, }, E_{I_{A}-\left\{Q_{1}\right\}} & =\frac{1}{4 \pi \varepsilon a^{2}} \cdot \frac{a}{\{2(D+\delta)-a\}} \cdot\left[-\sqrt{2} a s \sigma_{0} \pi e^{\frac{D^{2}}{2 s^{2}}}\left\{\Gamma\left(\frac{1}{2}, \frac{D^{2}}{2 s^{2}}\right)-\Gamma\left(\frac{1}{2}, \frac{R^{2}+D^{2}}{2 s^{2}}\right)\right\}\right. \\
+\sqrt{2} a s \sigma_{0} \pi e^{\frac{\left(D+2 \delta \delta^{2}\right.}{2 s^{2}}} & \left.\left\{\Gamma\left(\frac{1}{2}, \frac{(D+2 \delta)^{2}}{2 s^{2}}\right)-\Gamma\left(\frac{1}{2}, \frac{b^{2}+(D+2 \delta)^{2}}{2 s^{2}}\right)\right\}\right]
\end{aligned}
$$

$\left[\because Q_{1}=q_{i_{1}}+q_{i_{2}}\right.$, and substituting expressions for $q_{i_{1}}$ and $q_{i_{2}}$ given by equations (26) and (27) respectively]

$$
\begin{aligned}
& \text { or, } E_{I_{A}-\left\{Q_{1}\right\}}=\frac{1}{4 \pi \varepsilon a^{2}} \cdot \frac{a}{\{2(D+\delta)-a\}} \\
& \times\left[\sqrt{2} a s \sigma_{0} \pi e^{\frac{\left(D+2 \delta^{2}\right)^{2}}{2 s^{2}}}\left\{\Gamma\left(\frac{1}{2}, \frac{(D+2 \delta)^{2}}{2 s^{2}}\right)-\Gamma\left(\frac{1}{2}, \frac{b^{2}+(D+2 \delta)^{2}}{2 s^{2}}\right)\right\}\right. \\
& \left.-\sqrt{2} a s \sigma_{0} \pi e^{\frac{D^{2}}{2 s^{2}}}\left\{\Gamma\left(\frac{1}{2}, \frac{D^{2}}{2 s^{2}}\right)-\Gamma\left(\frac{1}{2}, \frac{R^{2}+D^{2}}{2 s^{2}}\right)\right\}\right] \\
& \text { or, } E_{I_{A}-\left\{Q_{1}\right\}}=\frac{1}{4 \pi \varepsilon a^{2}} \cdot \frac{a}{\{2(D+\delta)-a\}} \cdot \sqrt{2} a s \sigma_{0} \pi \\
& \times\left[e^{\frac{(D+2 \delta)^{2}}{2 s^{2}}}\left\{\Gamma\left(\frac{1}{2}, \frac{(D+2 \delta)^{2}}{2 s^{2}}\right)-\Gamma\left(\frac{1}{2}, \frac{b^{2}+(D+2 \delta)^{2}}{2 s^{2}}\right)\right\}\right. \\
& \left.-e^{\frac{D^{2}}{2 s^{2}}}\left\{\Gamma\left(\frac{1}{2}, \frac{D^{2}}{2 s^{2}}\right)-\Gamma\left(\frac{1}{2}, \frac{R^{2}+D^{2}}{2 s^{2}}\right)\right\}\right] \\
& \text { or, } E_{I_{A}-\left\{Q_{1}\right\}}=\frac{\sigma_{0}}{2 \sqrt{2} \varepsilon} \cdot\left(\frac{s}{a}\right) \cdot \frac{a}{\{2(D+\delta)-a\}} \cdot\left[e^{\frac{(D+2 \delta)^{2}}{2 s^{2}}}\left\{\Gamma\left(\frac{1}{2}, \frac{(D+2 \delta)^{2}}{2 s^{2}}\right)-\Gamma\left(\frac{1}{2}, \frac{b^{2}+(D+2 \delta)^{2}}{2 s^{2}}\right)\right\}\right. \\
& \left.-e^{\frac{D^{2}}{2 s^{2}}}\left\{\Gamma\left(\frac{1}{2}, \frac{D^{2}}{2 s^{2}}\right)-\Gamma\left(\frac{1}{2}, \frac{R^{2}+D^{2}}{2 s^{2}}\right)\right\}\right]
\end{aligned}
$$


or, $E_{I_{A}-\left\{\left\{_{1}\right\}\right.}=\frac{\sigma_{0}}{2 \sqrt{2} \varepsilon} \cdot\left(\frac{s}{a}\right) \cdot \frac{a}{\{2(D+\delta)-a\}} \cdot\left[e^{\frac{(D+2 \delta)^{2}}{2 s^{2}}}\left\{\Gamma\left(\frac{1}{2}, \frac{(D+2 \delta)^{2}}{2 s^{2}}\right)-\Gamma\left(\frac{1}{2}, \frac{(D+2 \delta)^{2}+b^{2}}{2 s^{2}}\right)\right\}\right.$ $\left.-e^{\frac{D^{2}}{2 s^{2}}}\left\{\Gamma\left(\frac{1}{2}, \frac{D^{2}}{2 s^{2}}\right)-\Gamma\left(\frac{1}{2}, \frac{D^{2}+R^{2}}{2 s^{2}}\right)\right\}\right]$

$E_{I_{o^{\prime}}}=\frac{1}{4 \pi \varepsilon\{2(D+\delta)-a\}^{2}} \cdot\left[-Q_{1}-\frac{a}{2(D+\delta)} \cdot Q_{1}-\frac{a^{2}}{\{2(D+\delta)\}^{2}} \cdot Q_{1}-\frac{a^{3}}{\{2(D+\delta)\}^{3}} \cdot Q_{1}\right.$

$-\cdots$ to $\infty$ terms]

$$
\begin{aligned}
\text { or, } E_{I_{o^{\prime}}} & =-\frac{Q_{1}}{4 \pi \varepsilon\{2(D+\delta)-a\}^{2}} \cdot\left[1+\frac{a}{2(D+\delta)}+\frac{a^{2}}{\{2(D+\delta)\}^{2}}+\frac{a^{3}}{\{2(D+\delta)\}^{3}}+\cdots \text { to } \infty \text { terms }\right] \\
& =-\frac{Q_{1}}{4 \pi \varepsilon\{2(D+\delta)-a\}^{2}} \cdot \frac{1}{\left\{1-\frac{a}{2(D+\delta)}\right\}} \\
& =-\frac{Q_{1}}{4 \pi \varepsilon\{2(D+\delta)-a\}^{2}} \cdot \frac{2(D+\delta)}{\{2(D+\delta)-a\}}
\end{aligned}
$$

or, $E_{I_{o}^{\prime}}=-\frac{1}{4 \pi \varepsilon\{2(D+\delta)-a\}^{2}} \cdot\left[\frac{2(D+\delta)}{\{2(D+\delta)-a\}} \cdot Q_{1}\right] \ldots$

$o r, E_{I_{o^{\prime}}}=-\frac{1}{4 \pi \varepsilon\{2(D+\delta)-a\}^{2}} \cdot \frac{2(D+\delta)}{\{2(D+\delta)-a\}} \cdot\left[-\sqrt{2} a s \sigma_{0} \pi e^{\frac{D^{2}}{2 s^{2}}}\left\{\Gamma\left(\frac{1}{2}, \frac{D^{2}}{2 s^{2}}\right)-\Gamma\left(\frac{1}{2}, \frac{R^{2}+D^{2}}{2 s^{2}}\right)\right\}\right.$

$\left.+\sqrt{2} a s \sigma_{0} \pi e^{\frac{\left.(D+2)^{2}\right)^{2}}{2 s^{2}}}\left\{\Gamma\left(\frac{1}{2}, \frac{(D+2 \delta)^{2}}{2 s^{2}}\right)-\Gamma\left(\frac{1}{2}, \frac{b^{2}+(D+2 \delta)^{2}}{2 s^{2}}\right)\right\}\right]$

$\left[\because Q_{1}=q_{i_{1}}+q_{i_{2}}\right.$, and substituting expressions for $q_{i_{1}}$ and $q_{i_{2}}$ given by equations (26) and (27) respectively]

$$
\begin{aligned}
o r, E_{I_{o}}=-\frac{1}{4 \pi \varepsilon\{2(D+\delta)-a\}^{2}} \cdot \frac{2(D+\delta)}{\{2(D+\delta)-a\}} \cdot \sqrt{2} a s \sigma_{0} \pi \\
\times\left[e^{\frac{\left(\frac{D+2 \delta)^{2}}{2 s^{2}}\right.}{2(D)}}\left\{\Gamma\left(\frac{1}{2}, \frac{(D+2 \delta)^{2}}{2 s^{2}}\right)-\Gamma\left(\frac{1}{2}, \frac{b^{2}+(D+2 \delta)^{2}}{2 s^{2}}\right)\right\}\right. \\
\left.\quad-e^{\frac{D^{2}}{2 s^{2}}}\left\{\Gamma\left(\frac{1}{2}, \frac{D^{2}}{2 s^{2}}\right)-\Gamma\left(\frac{1}{2}, \frac{R^{2}+D^{2}}{2 s^{2}}\right)\right\}\right]
\end{aligned}
$$




$$
\text { or, } \begin{aligned}
E_{I_{o^{\prime}}}=-\frac{\sigma_{0}}{2 \sqrt{2} \varepsilon} & \cdot\left(\frac{s}{a}\right) \cdot\left[\frac{a}{\{2(D+\delta)-a\}}\right]^{2} \cdot\left[\frac{2(D+\delta)}{\{2(D+\delta)-a\}}\right] \\
\times & {\left[e^{\frac{(D+2 \delta)^{2}}{2 s^{2}}}\left\{\Gamma\left(\frac{1}{2}, \frac{(D+2 \delta)^{2}}{2 s^{2}}\right)-\Gamma\left(\frac{1}{2}, \frac{(D+2 \delta)^{2}+b^{2}}{2 s^{2}}\right)\right\}\right.} \\
& \left.-e^{\frac{D^{2}}{2 s^{2}}}\left\{\Gamma\left(\frac{1}{2}, \frac{D^{2}}{2 s^{2}}\right)-\Gamma\left(\frac{1}{2}, \frac{D^{2}+R^{2}}{2 s^{2}}\right)\right\}\right]
\end{aligned}
$$

We now have expressions for all the terms of $E_{P, n e t}$ given by equation (20) which equation is reproduced below for convenience.

$$
E_{P, \text { net }}=E_{B, q_{i_{1}}}+E_{B^{\prime}, q_{i_{2}}}+E_{I_{A}-\left\{Q_{1}\right\}}+E_{I_{o^{\prime}}} \ldots(20),
$$

where $E_{B, q_{11}}$ is given by equation (21), $E_{B^{\prime}, q_{i 2}}$ is given by equation (22), $E_{I_{A}-\left\{Q_{1}\right\}}$ is given by equation (29) and by equation (30), and $E_{I_{o}}$ is given by equation (32) and by equation (33). These equations are reproduced below for convenience.

$$
\begin{aligned}
& E_{B, q_{i 1}}=\frac{\sigma_{0}}{2 \varepsilon a}\left[\frac{\left\{s^{2}-2 a(D-a)\right\}}{\sqrt{2} s} \cdot e^{\frac{(D-a)^{2}}{2 s^{2}}}\left\{\Gamma\left(\frac{1}{2}, \frac{(D-a)^{2}}{2 s^{2}}\right)-\Gamma\left(\frac{1}{2}, \frac{(D-a)^{2}+R^{2}}{2 s^{2}}\right)\right\}\right. \\
& \left.+2 a\left\{1-\frac{(D-a)}{\sqrt{(D-a)^{2}+R^{2}}} \cdot e^{-\frac{R^{2}}{2 s^{2}}}\right\}\right]=\frac{\sigma_{0}}{2 \varepsilon a} \cdot \phi(D, a, R, s) \\
& E_{B^{\prime}, q_{i_{2}}}=-\frac{\sigma_{0}}{2 \varepsilon a}\left[\frac{\left\{s^{2}-2 a(\overline{D+2 \delta}-a)\right\}}{\sqrt{2} s} \cdot e^{\frac{(\overline{D+2 \delta}-a)^{2}}{2 s^{2}}}\left\{\Gamma\left(\frac{1}{2}, \frac{(\overline{D+2 \delta}-a)^{2}}{2 s^{2}}\right)-\Gamma\left(\frac{1}{2}, \frac{(\overline{D+2 \delta}-a)^{2}+b^{2}}{2 s^{2}}\right)\right\}\right. \\
& +2 a\left\{1-\frac{(\overline{D+2 \delta}-a)}{\sqrt{(\overline{D+2 \delta}-a)^{2}+b^{2}}} \cdot e^{-\frac{b^{2}}{2 s^{2}}}\right\}=-\frac{\sigma_{0}}{2 \varepsilon a} \cdot \phi(\overline{D+2 \delta}, a, b, s)
\end{aligned}
$$

$$
\begin{aligned}
& E_{I_{A}-\left\{Q_{1}\right\}}=\frac{1}{4 \pi \varepsilon a^{2}} \cdot\left[\frac{a}{\{2(D+\delta)-a\}} \cdot Q_{1}\right] \ldots \text { (29) } \\
& o r, E_{I_{A}-\left\{Q_{1}\right\}}=\frac{\sigma_{0}}{2 \sqrt{2} \varepsilon} \cdot\left(\frac{s}{a}\right) \cdot \frac{a}{\{2(D+\delta)-a\}} \cdot\left[e^{\frac{(D+2 \delta)^{2}}{2 s^{2}}}\left\{\Gamma\left(\frac{1}{2}, \frac{(D+2 \delta)^{2}}{2 s^{2}}\right)-\Gamma\left(\frac{1}{2}, \frac{(D+2 \delta)^{2}+b^{2}}{2 s^{2}}\right)\right\}\right. \\
& \left.-e^{\frac{D^{2} s^{2}}{2}}\left\{\Gamma\left(\frac{1}{2}, \frac{D^{2}}{2 s^{2}}\right)-\Gamma\left(\frac{1}{2}, \frac{D^{2}+R^{2}}{2 s^{2}}\right)\right\}\right]
\end{aligned}
$$




$$
\begin{aligned}
& E_{I_{o^{\prime}}}=-\frac{1}{4 \pi \varepsilon\{2(D+\delta)-a\}^{2}} \cdot\left[\frac{2(D+\delta)}{\{2(D+\delta)-a\}} \cdot Q_{1}\right] \ldots \\
& \text { or, } E_{I_{o^{\prime}}}=-\frac{\sigma_{0}}{2 \sqrt{2} \varepsilon} \cdot\left(\frac{s}{a}\right) \cdot\left[\frac{a}{\{2(D+\delta)-a\}}\right]^{2} \cdot\left[\frac{2(D+\delta)}{\{2(D+\delta)-a\}}\right] \\
& \times\left[e^{\frac{(D+2 \delta)^{2}}{2 s^{2}}}\left\{\Gamma\left(\frac{1}{2}, \frac{(D+2 \delta)^{2}}{2 s^{2}}\right)-\Gamma\left(\frac{1}{2}, \frac{(D+2 \delta)^{2}+b^{2}}{2 s^{2}}\right)\right\}\right. \\
& \left.-e^{\frac{D^{2}}{s^{2}}}\left\{\Gamma\left(\frac{1}{2}, \frac{D^{2}}{2 s^{2}}\right)-\Gamma\left(\frac{1}{2}, \frac{D^{2}+R^{2}}{2 s^{2}}\right)\right\}\right]
\end{aligned}
$$

We have, thus, found our expression (by far the most accurate) for the electric field produced at the point $\mathrm{P}$, the point of the earthed sphere in the system of our interest (the system constituted by an insulating disk of which one surface has charges on it and the surface opposite to it has on it an earthed metal disk and an earthed metal sphere approaching the surface of the disk on which charges are present as shown in fig. 2) which point is nearest to the charge surface on the insulating disk for a charge distribution profile on the surface of the insulating disk which has charges on it given by

$$
\sigma(r, \theta)=\sigma_{0} e^{-r^{2} / 2 s^{2}}
$$

where $\sigma_{0}$ is the maximum density of charge on the insulator and $s$ is a parameter which controls the width of the "bell" of this bell-shaped charge distribution. We write this expression for convenience as follows. 


$$
\begin{aligned}
& E_{P, \text { net }}=\frac{\sigma_{0}}{2 \varepsilon a}\left[\frac{\left\{s^{2}-2 a(D-a)\right\}}{\sqrt{2} s} \cdot e^{\frac{(D-a)^{2}}{2 s^{2}}}\left\{\Gamma\left(\frac{1}{2}, \frac{(D-a)^{2}}{2 s^{2}}\right)-\Gamma\left(\frac{1}{2}, \frac{(D-a)^{2}+R^{2}}{2 s^{2}}\right)\right\}\right. \\
& \left.+2 a\left\{1-\frac{(D-a)}{\sqrt{(D-a)^{2}+R^{2}}} \cdot e^{-\frac{R^{2}}{2 s^{2}}}\right\}\right] \\
& -\frac{\sigma_{0}}{2 \varepsilon a}\left[\frac{\left\{s^{2}-2 a(\overline{D+2 \delta}-a)\right\}}{\sqrt{2} s} \cdot e^{\frac{(\overline{D+2 \delta}-a)^{2}}{2 s^{2}}}\left\{\Gamma\left(\frac{1}{2}, \frac{(\overline{D+2 \delta}-a)^{2}}{2 s^{2}}\right)-\Gamma\left(\frac{1}{2}, \frac{(\overline{D+2 \delta}-a)^{2}+b^{2}}{2 s^{2}}\right)\right\}\right. \\
& \left.+2 a\left\{1-\frac{(\overline{D+2 \delta}-a)}{\sqrt{(\overline{D+2 \delta}-a)^{2}+b^{2}}} \cdot e^{-\frac{b^{2}}{2 s^{2}}}\right\}\right] \\
& +\frac{\sigma_{0}}{2 \sqrt{2} \varepsilon} \cdot\left(\frac{s}{a}\right) \cdot \frac{a}{\{2(D+\delta)-a\}} \cdot\left[e^{\frac{(D+2 \delta)^{2}}{2 s^{2}}}\left\{\Gamma\left(\frac{1}{2}, \frac{(D+2 \delta)^{2}}{2 s^{2}}\right)-\Gamma\left(\frac{1}{2}, \frac{(D+2 \delta)^{2}+b^{2}}{2 s^{2}}\right)\right\}\right. \\
& \left.-e^{\frac{D^{2}}{2 s^{2}}}\left\{\Gamma\left(\frac{1}{2}, \frac{D^{2}}{2 s^{2}}\right)-\Gamma\left(\frac{1}{2}, \frac{D^{2}+R^{2}}{2 s^{2}}\right)\right\}\right] \\
& -\frac{\sigma_{0}}{2 \sqrt{2} \varepsilon} \cdot\left(\frac{s}{a}\right) \cdot\left[\frac{a}{\{2(D+\delta)-a\}}\right]^{2} \cdot\left[\frac{2(D+\delta)}{\{2(D+\delta)-a\}}\right] \\
& \times\left[e^{\frac{(D+2 \delta)^{2}}{2 s^{2}}}\left\{\Gamma\left(\frac{1}{2}, \frac{(D+2 \delta)^{2}}{2 s^{2}}\right)-\Gamma\left(\frac{1}{2}, \frac{(D+2 \delta)^{2}+b^{2}}{2 s^{2}}\right)\right\}\right. \\
& \left.-e^{\frac{D^{2}}{2 s^{2}}}\left\{\Gamma\left(\frac{1}{2}, \frac{D^{2}}{2 s^{2}}\right)-\Gamma\left(\frac{1}{2}, \frac{D^{2}+R^{2}}{2 s^{2}}\right)\right\}\right]
\end{aligned}
$$




$$
\begin{gathered}
\text { or, } E_{P, n e t}=\frac{\sigma_{0}}{2 \varepsilon}\left[\frac{\left\{s^{2}-2 a(D-a)\right\}}{\sqrt{2} s a} \cdot e^{\frac{(D-a)^{2}}{2 s^{2}}}\left\{\Gamma\left(\frac{1}{2}, \frac{(D-a)^{2}}{2 s^{2}}\right)-\Gamma\left(\frac{1}{2}, \frac{(D-a)^{2}+R^{2}}{2 s^{2}}\right)\right\}\right. \\
+2\left\{1-\frac{(D-a)}{\sqrt{(D-a)^{2}+R^{2}}} \cdot e^{-\frac{R^{2}}{s^{2}}}\right\} \\
-\frac{\left\{s^{2}-2 a(\overline{D+2 \delta}-a)\right\}}{\sqrt{2} s a} \cdot e^{\frac{(\overline{D+2 \delta}-a)^{2}}{2 s^{2}}}\left\{\Gamma\left(\frac{1}{2}, \frac{(\overline{D+2 \delta}-a)^{2}}{2 s^{2}}\right)-\Gamma\left(\frac{1}{2}, \frac{(\overline{D+2 \delta}-a)^{2}+b^{2}}{2 s^{2}}\right)\right\} \\
-2\left\{1-\frac{(\overline{D+2 \delta}-a)}{\sqrt{(\overline{D+2 \delta}-a)^{2}+b^{2}}} \cdot e^{-\frac{b^{2}}{2 s^{2}}}\right\} \\
+\frac{1}{\sqrt{2}} \cdot\left(\frac{s}{a}\right) \cdot \frac{a}{\{2(D+\delta)-a\}} \cdot\left\{e^{\frac{(D+2 \delta)^{2}}{2 s^{2}}}\left\{\Gamma\left(\frac{1}{2}, \frac{(D+2 \delta)^{2}}{2 s^{2}}\right)-\Gamma\left(\frac{1}{2}, \frac{(D+2 \delta)^{2}+b^{2}}{2 s^{2}}\right)\right\}\right. \\
-\frac{1}{\sqrt{2}} \cdot\left(\frac{s}{a}\right) \cdot\left\{\frac{a}{\{2(D+\delta)-a\}}\right\} \cdot\left\{\frac{2(D+\delta)}{\{2(D+\delta)-a\}}\right\} \\
\quad \times\left\{e^{\frac{D^{2} s^{2}}{2\left(D+2 s^{2}\right.}}\left\{\Gamma\left(\frac{1}{2}, \frac{D^{2}}{2 s^{2}}\right)-\Gamma\left(\frac{1}{2}, \frac{D^{2}+R^{2}}{2 s^{2}}\right)\right\}\right\} \\
\quad-e^{\frac{D^{2} s^{2}}{2 s^{2}}}\left\{\Gamma\left(\frac{1}{2}, \frac{(D+2 \delta)^{2}}{2 s^{2}}\right)-\Gamma\left(\frac{1}{2}, \frac{(D+2 \delta)^{2}+b^{2}}{2 s^{2}}\right)\right\} \\
\left.\left.\left.\left.2 s^{2}\right)-\Gamma\left(\frac{1}{2}, \frac{D^{2}+R^{2}}{2 s^{2}}\right)\right\}\right\}\right]
\end{gathered}
$$

or, $E_{P, \text { net }}=\frac{\sigma_{0}}{2 \varepsilon} f_{1}(D, \delta, a, R, b, s) \cdots(36)$ 
where

$$
\begin{aligned}
& f_{1}(D, \delta, a, R, b, s)=\left[\frac{\left\{s^{2}-2 a(D-a)\right\}}{\sqrt{2} s a} \cdot e^{\frac{(D-a)^{2}}{2 s^{2}}}\left\{\Gamma\left(\frac{1}{2}, \frac{(D-a)^{2}}{2 s^{2}}\right)-\Gamma\left(\frac{1}{2}, \frac{(D-a)^{2}+R^{2}}{2 s^{2}}\right)\right\}\right. \\
& +2\left\{1-\frac{(D-a)}{\sqrt{(D-a)^{2}+R^{2}}} \cdot e^{-\frac{R^{2}}{2 s^{2}}}\right\} \\
& -\frac{\left\{s^{2}-2 a(\overline{D+2 \delta}-a)\right\}}{\sqrt{2} s a} \cdot e^{\frac{(\overline{D+2 \delta}-a)^{2}}{2 s^{2}}}\left\{\Gamma\left(\frac{1}{2}, \frac{(\overline{D+2 \delta}-a)^{2}}{2 s^{2}}\right)-\Gamma\left(\frac{1}{2}, \frac{(\overline{D+2 \delta}-a)^{2}+b^{2}}{2 s^{2}}\right)\right\} \\
& -2\left\{1-\frac{(\overline{D+2 \delta}-a)}{\sqrt{(\overline{D+2 \delta}-a)^{2}+b^{2}}} \cdot e^{-\frac{b^{2}}{2 s^{2}}}\right\} \\
& +\frac{1}{\sqrt{2}} \cdot\left(\frac{s}{a}\right) \cdot \frac{a}{\{2(D+\delta)-a\}} \cdot\left\{e^{\frac{(D+2 \delta)^{2}}{2 s^{2}}}\left\{\Gamma\left(\frac{1}{2}, \frac{(D+2 \delta)^{2}}{2 s^{2}}\right)-\Gamma\left(\frac{1}{2}, \frac{(D+2 \delta)^{2}+b^{2}}{2 s^{2}}\right)\right\}\right. \\
& \left.-e^{\frac{D^{2}}{2 s^{2}}}\left\{\Gamma\left(\frac{1}{2}, \frac{D^{2}}{2 s^{2}}\right)-\Gamma\left(\frac{1}{2}, \frac{D^{2}+R^{2}}{2 s^{2}}\right)\right\}\right\} \\
& -\frac{1}{\sqrt{2}} \cdot\left(\frac{s}{a}\right) \cdot\left\{\frac{a}{\{2(D+\delta)-a\}}\right\}^{2} \cdot\left\{\frac{2(D+\delta)}{\{2(D+\delta)-a\}}\right\} \\
& \times\left\{e^{\frac{(D+2 \delta)^{2}}{2 s^{2}}}\left\{\Gamma\left(\frac{1}{2}, \frac{(D+2 \delta)^{2}}{2 s^{2}}\right)-\Gamma\left(\frac{1}{2}, \frac{(D+2 \delta)^{2}+b^{2}}{2 s^{2}}\right)\right\}\right. \\
& \left.\left.-e^{\frac{D^{2}}{2 s^{2}}}\left\{\Gamma\left(\frac{1}{2}, \frac{D^{2}}{2 s^{2}}\right)-\Gamma\left(\frac{1}{2}, \frac{D^{2}+R^{2}}{2 s^{2}}\right)\right\}\right\}\right]
\end{aligned}
$$

\section{To Find the Minimum Value of $\sigma_{0}$ Required for a Discharge to Occur at the Point of the Earthed Metal Sphere Nearest to the Charged Surface of the Insulating Disk}

To find the minimum value of $\sigma_{0}$ required for a discharge to occur at the point of the earthed metal sphere nearest to the charged surface of the insulating disk, we need to put the value of the breakdown field for $E_{P, n e t}$ and also put $D=a$ in equation (35). Then, we have 


$$
\begin{gathered}
E_{\text {brakkdown }}=\frac{\sigma_{0}}{2 \varepsilon}\left[\frac{s^{2}}{\sqrt{2} s a} \cdot\left\{\Gamma\left(\frac{1}{2}, 0\right)-\Gamma\left(\frac{1}{2}, \frac{R^{2}}{2 s^{2}}\right)\right\}+2\right. \\
-\frac{\left\{s^{2}-4 a \delta\right\}}{\sqrt{2} s a} \cdot e^{\frac{4 \delta^{2}}{2 s^{2}}}\left\{\Gamma\left(\frac{1}{2}, \frac{4 \delta^{2}}{2 s^{2}}\right)-\Gamma\left(\frac{1}{2}, \frac{4 \delta^{2}+b^{2}}{2 s^{2}}\right)\right\}-2\left\{1-\frac{2 \delta}{\sqrt{4 \delta^{2}+b^{2}}} \cdot e^{-\frac{b^{2}}{2 s^{2}}}\right\} \\
+\frac{1}{\sqrt{2}} \cdot\left(\frac{s}{a}\right) \cdot \frac{a}{\{2 \delta+a\}} \cdot\left\{e^{\frac{(a+2 s)^{2}}{2 s^{2}}}\left\{\Gamma\left(\frac{1}{2}, \frac{(a+2 \delta)^{2}}{2 s^{2}}\right)-\Gamma\left(\frac{1}{2}, \frac{(a+2 \delta)^{2}+b^{2}}{2 s^{2}}\right)\right\}\right. \\
-\frac{1}{\sqrt{2}} \cdot\left(\frac{s}{a}\right) \cdot\left\{\frac{a}{(2 \delta+a)}\right\}^{2} \cdot\left\{\frac{e^{\frac{2}{2 s^{2}}}}{(2(a+\delta)}\left(\Gamma\left(\frac{1}{2}, \frac{a^{2}}{2 s^{2}}\right)-\Gamma\left(\frac{1}{2}, \frac{a^{2}+R^{2}}{2 s^{2}}\right)\right\}\right\} \\
\times\left\{e^{\frac{(2 \delta+a t a)^{2}}{2 s^{2}}}\left\{\Gamma\left(\frac{1}{2}, \frac{(a+2 \delta)^{2}}{2 s^{2}}\right)-\Gamma\left(\frac{1}{2}, \frac{(a+2 \delta)^{2}+b^{2}}{2 s^{2}}\right)\right\}\right. \\
\left.\left.-e^{\frac{a^{2}}{2 s^{2}}}\left\{\Gamma\left(\frac{1}{2}, \frac{a^{2}}{2 s^{2}}\right)-\Gamma\left(\frac{1}{2}, \frac{a^{2}+R^{2}}{2 s^{2}}\right)\right\}\right\}\right]
\end{gathered}
$$

$$
\begin{gathered}
\text { or, } E_{\text {breakdown }}=\frac{\sigma_{0}}{2 \varepsilon}\left[\frac{s}{\sqrt{2} a} \cdot\left\{\Gamma\left(\frac{1}{2}, 0\right)-\Gamma\left(\frac{1}{2}, \frac{R^{2}}{2 s^{2}}\right)\right\}+2\right. \\
-\frac{\left\{s^{2}-4 a \delta\right\}}{\sqrt{2} s a} \cdot e^{\frac{2 \delta^{2}}{s^{2}}}\left\{\Gamma\left(\frac{1}{2}, \frac{2 \delta^{2}}{s^{2}}\right)-\Gamma\left(\frac{1}{2}, \frac{4 \delta^{2}+b^{2}}{2 s^{2}}\right)\right\}-2\left\{1-\frac{2 \delta}{\sqrt{4 \delta^{2}+b^{2}}} \cdot e^{-\frac{b^{2}}{2 s^{2}}}\right\} \\
+\frac{1}{\sqrt{2}} \cdot\left(\frac{s}{a}\right) \cdot \frac{a}{\{2 \delta+a\}} \cdot\left\{e^{\frac{(a+2 \delta)^{2}}{2 s^{2}}}\left\{\Gamma\left(\frac{1}{2}, \frac{(a+2 \delta)^{2}}{2 s^{2}}\right)-\Gamma\left(\frac{1}{2}, \frac{(a+2 \delta)^{2}+b^{2}}{2 s^{2}}\right)\right\}\right. \\
-\frac{1}{\sqrt{2}} \cdot\left(\frac{s}{a}\right) \cdot\left\{\frac{a}{(2 \delta+a)}\right\}^{2} \cdot\left\{\frac{2(a+\delta)}{(2 \delta+a)}\right\} \\
\quad \times\left\{e^{\frac{(2 \delta+a)^{2}}{2 s^{2}}}\left\{\Gamma\left(\frac{a^{2}}{2}, \frac{(a+2 \delta)^{2}}{2 s^{2}}\right)-\Gamma\left(\frac{1}{2}, \frac{(a+2 \delta)^{2}+b^{2}}{2 s^{2}}\right)\right\}\right. \\
\left.-e^{\frac{a^{2}}{2 s^{2}}}\left\{\Gamma\left(\frac{a^{2}}{2 s^{2}}\right)-\Gamma\left(\frac{1}{2}, \frac{a^{2}+R^{2}}{2 s^{2}}\right)\right\}\right\} \\
\left.\left.\left.\left.2 s^{2}\right)-\Gamma\left(\frac{1}{2}, \frac{a^{2}+R^{2}}{2 s^{2}}\right)\right\}\right\}\right]
\end{gathered}
$$


or,$E_{\text {breakdown }}=\frac{\sigma_{0}}{2 \varepsilon} f_{2}(\delta, a, R, b, s) \ldots(40)$

where

$$
\begin{gathered}
f_{2}(\delta, a, R, b, s)=\left[\frac{s}{\sqrt{2} a} \cdot\left\{\Gamma\left(\frac{1}{2}, 0\right)-\Gamma\left(\frac{1}{2}, \frac{R^{2}}{2 s^{2}}\right)\right\}+2\right. \\
-\frac{\left\{s^{2}-4 a \delta\right\}}{\sqrt{2} s a} \cdot e^{\frac{2 \delta^{2}}{s^{2}}}\left\{\Gamma\left(\frac{1}{2}, \frac{2 \delta^{2}}{s^{2}}\right)-\Gamma\left(\frac{1}{2}, \frac{4 \delta^{2}+b^{2}}{2 s^{2}}\right)\right\}-2\left\{1-\frac{2 \delta}{\sqrt{4 \delta^{2}+b^{2}}} \cdot e^{-\frac{b^{2}}{2 s^{2}}}\right\} \\
+\frac{1}{\sqrt{2}} \cdot\left(\frac{s}{a}\right) \cdot \frac{a}{\{2 \delta+a\}} \cdot\left\{e^{\frac{(a+2 \delta)^{2}}{2 s^{2}}}\left\{\Gamma\left(\frac{1}{2}, \frac{(a+2 \delta)^{2}}{2 s^{2}}\right)-\Gamma\left(\frac{1}{2}, \frac{(a+2 \delta)^{2}+b^{2}}{2 s^{2}}\right)\right\}\right. \\
-\frac{1}{\sqrt{2}} \cdot\left(\frac{s}{a}\right) \cdot\left\{\frac{a}{(2 \delta+a)}\right\}^{2} \cdot\left\{\frac{2(a+\delta)}{(2 \delta+a)}\right\} \\
\times\left\{e^{\frac{a^{2}}{2 s^{2}}}\left\{\Gamma\left(\frac{1}{2}, \frac{a^{2}}{2 s^{2}}\right)-\Gamma\left(\frac{1}{2}, \frac{a^{2}+R^{2}}{2 s^{2}}\right)\right\}\right\} \\
\left.\left.\quad-e^{\frac{a^{2}}{2 s^{2}}}\left\{\Gamma\left(\frac{1}{2}, \frac{a^{2}}{2 s^{2}}\right)-\Gamma\left(\frac{1}{2}, \frac{(a+2 \delta)^{2}}{2 s^{2}}\right)-\Gamma\left(\frac{1}{2}, \frac{(a+2 \delta)^{2}+b^{2}}{2 s^{2}}\right)\right\}\right\}\right]
\end{gathered}
$$

or, $\sigma_{0}=2 \varepsilon E_{\text {breakdown }}\left[f_{2}(\delta, a, R, b, s)\right]^{-1} \ldots(42)$

Equation (42) used with equation (41), thus, gives us the minimum value of $\sigma_{0}$ required for a discharge to occur.

We may also find the minimum value of $\sigma_{0}$ required for a discharge to occur at the point of the earthed metal sphere nearest to the charged surface of the insulating disk when its centre is at a distance $D$ from the charged surface of the insulating disk. This value can be found by putting the value of the breakdown electric field in equation (36). On doing so, we have

$\left.\sigma_{0}\right|_{\min }(D, \delta, a, R, b, s)=\frac{2 \varepsilon E_{\text {breakdown }}}{f_{1}(D, \delta, a, R, b, s)} \cdots(42 A)$,

where $f_{1}(D, \delta, a, R, b, s)$ is given by equation (37). 


\section{To Find the Electric Field at a Point Inside the Insulating Disk on the $z$-axis:}

We observe that the electric field inside the insulating disk is maximum at points on the $z$ axis as these points are nearest to the centre of the charged surface of the insulating disk where the density of charge is maximum as this centre is the origin of our Cartesian coordinate system. Hence, we find here the electric field at a point inside the insulating disk on the z-axis. We first find out the expression for the electric field at a point $(0,0, z)$ due only to the charge on the charged surface of the insulator, i.e., to the surface of charge B shown in Fig. 4. The $x, y$ and $z$ components of the electric field at a point $(0,0, z)$ due to an element of charge at a point $(r, \theta)$ having a charge $\sigma(r, \theta) \cdot r d r d \theta$ on the surface of charge B are as follows:

$$
\begin{aligned}
& d E_{x}=\frac{\sigma(r, \theta) \cdot r d r d \theta}{4 \pi \varepsilon\left(r^{2}+z^{2}\right)} \cdot \frac{r}{\sqrt{r^{2}+z^{2}}} \cdot \cos \theta=\frac{\sigma_{0} e^{-\frac{r^{2}}{2 s^{2}}} \cdot r^{2} \cos \theta \cdot d r d \theta}{4 \pi \varepsilon\left(r^{2}+z^{2}\right)^{\frac{3}{2}}} \cdots(43), \\
& d E_{y}=\frac{\sigma(r, \theta) \cdot r d r d \theta}{4 \pi \varepsilon\left(r^{2}+z^{2}\right)} \cdot \frac{r}{\sqrt{r^{2}+z^{2}}} \cdot \sin \theta=\frac{\sigma_{0} e^{-\frac{r^{2}}{2 s^{2}}} \cdot r^{2} \sin \theta \cdot d r d \theta}{4 \pi \varepsilon\left(r^{2}+z^{2}\right)^{\frac{3}{2}}} \cdots(44), \text { and } \\
& d E_{z}=\frac{\sigma(r, \theta) \cdot r d r d \theta}{4 \pi \varepsilon\left(r^{2}+z^{2}\right)} \cdot \frac{z}{\sqrt{r^{2}+z^{2}}}=\frac{\sigma_{0} e^{-\frac{r^{2}}{2 s^{2}}} \cdot r z \cdot d r d \theta}{4 \pi \varepsilon\left(r^{2}+z^{2}\right)^{\frac{3}{2}}} \cdots(45) .
\end{aligned}
$$

Therefore, the $x, y$ and $z$ components of the electric field at the point $(0,0, z)$ due to the surface of charge B are as follows:

$$
\begin{aligned}
& E_{x}=\int_{0}^{2 \pi} \int_{0}^{R} \frac{\sigma_{0} e^{-\frac{r^{2}}{2 s^{2}}} \cdot r^{2} \cos \theta \cdot d r d \theta}{4 \pi \varepsilon\left(r^{2}+z^{2}\right)^{\frac{3}{2}}} \cdots(46) \\
& \text { or, } E_{x}=\frac{\sigma_{0}}{4 \pi \varepsilon} \cdot \int_{0}^{2 \pi} \cos \theta d \theta \cdot \int_{0}^{R} \frac{e^{-\frac{r^{2}}{2 s^{2}}} \cdot r^{2} d r}{\left(r^{2}+z^{2}\right)^{\frac{3}{2}}} \\
& \text { or, } E_{x}=\frac{\sigma_{0}}{4 \pi \varepsilon} \cdot[\sin \theta]_{0}^{2 \pi} \cdot \int_{0}^{R} \frac{e^{-\frac{r^{2}}{2 s^{2}}} \cdot r^{2} d r}{\left(r^{2}+z^{2}\right)^{\frac{3}{2}}} \\
& \text { or, } E_{x}=0 \cdots(47) \\
& E_{y}=\int_{0}^{2 \pi} \int_{0}^{R} \frac{\sigma_{0} e^{-\frac{r^{2}}{2 s^{2}}} \cdot r^{2} \sin \theta \cdot d r d \theta}{4 \pi \varepsilon\left(r^{2}+z^{2}\right)^{\frac{3}{2}}} \cdots(48) \\
& \text { or, } E_{y}=\frac{\sigma_{0}}{4 \pi \varepsilon} \cdot \int_{0}^{2 \pi} \sin \theta d \theta \cdot \int_{0}^{R} \frac{e^{-\frac{r^{2}}{2 s^{2}}} \cdot r^{2} d r}{\left(r^{2}+z^{2}\right)^{\frac{3}{2}}}
\end{aligned}
$$




$$
\begin{aligned}
& \text { or, } E_{y}=\frac{\sigma_{0}}{4 \pi \varepsilon} \cdot[-\cos \theta]_{0}^{2 \pi} \cdot \int_{0}^{R} \frac{e^{-\frac{r^{2}}{2 s^{2}}} \cdot r^{2} d r}{\left(r^{2}+z^{2}\right)^{\frac{3}{2}}} \\
& \text { or, } E_{y}=0 \text {. } \\
& E_{z}=\int_{0}^{2 \pi} \int_{0}^{R} \frac{\sigma_{0} e^{-\frac{r^{2}}{2 s^{2}}} \cdot r z \cdot d r d \theta}{4 \pi \varepsilon\left(r^{2}+z^{2}\right)^{\frac{3}{2}}} \\
& \text { or, } E_{z}=\frac{\sigma_{0} Z}{4 \pi \varepsilon} \int_{0}^{2 \pi} d \theta \int_{0}^{R} \frac{e^{-\frac{r^{2}}{2 s^{2}}} \cdot r d r}{\left(r^{2}+z^{2}\right)^{\frac{3}{2}}} \\
& \text { or }, E_{z}=\frac{\sigma_{0} z}{4 \pi \varepsilon} \cdot 2 \pi \cdot \frac{1}{2} \int_{r=0}^{r=R} \frac{e^{-\frac{r^{2}}{2 s^{2}}} \cdot d\left(r^{2}\right)}{\left(r^{2}+z^{2}\right)^{\frac{3}{2}}} \\
& \text { or }, E_{z}=\frac{\sigma_{0} Z}{4 \varepsilon} \int_{r=0}^{r=R} \frac{e^{-\frac{r^{2}}{2 s^{2}}} \cdot d\left(r^{2}\right)}{\left(r^{2}+z^{2}\right)^{\frac{3}{2}}} \\
& \text { or }, E_{z}=\frac{\sigma_{0} Z}{4 \varepsilon} \cdot 2 s^{2} \cdot \int_{r=0}^{r=R} \frac{e^{-\frac{r^{2}}{2 s^{2}}} \cdot d\left(\frac{r^{2}}{2 s^{2}}\right)}{\left[2 s^{2}\left(\frac{r^{2}}{2 s^{2}}\right)+z^{2}\right]^{\frac{3}{2}}} \\
& \text { or, } E_{z}=\frac{\sigma_{0} Z}{4 \varepsilon} \cdot 2 s^{2} \cdot \frac{1}{2 \sqrt{2} s^{3}} \int_{0}^{\frac{R^{2}}{2 s^{2}}} \frac{e^{-t} d t}{\left(t+\frac{z^{2}}{2 s^{2}}\right)^{\frac{3}{2}}}\left[\text { where } t=\frac{r^{2}}{2 s^{2}}\right] \\
& o r, E_{z}=\frac{\sigma_{0} z}{4 \sqrt{2} \varepsilon s} \int_{0}^{\frac{R^{2}}{2 s^{2}}} \frac{e^{-t} d t}{\left(t+\frac{z^{2}}{2 s^{2}}\right)^{\frac{3}{2}}} \\
& \text { or, } E_{z}=\frac{\sigma_{0} Z}{4 \sqrt{2} \varepsilon s} \cdot e^{\frac{z^{2}}{2 s^{2}}} \int_{0}^{\frac{R^{2}}{2 s^{2}}} \frac{e^{-\left(t+\frac{z^{2}}{2 s^{2}}\right)} d t}{\left(t+\frac{z^{2}}{2 s^{2}}\right)^{\frac{3}{2}}} \\
& \text { or }, E_{z}=\frac{\sigma_{0} Z}{4 \sqrt{2} \varepsilon s} \cdot e^{\frac{z^{2}}{2 s^{2}}} \int_{t=0}^{t=\frac{R^{2}}{2 s^{2}}}\left(t+\frac{z^{2}}{2 s^{2}}\right)^{-\frac{3}{2}} e^{-\left(t+\frac{z^{2}}{2 s^{2}}\right)} d\left(t+\frac{z^{2}}{2 s^{2}}\right) \\
& \text { or }, E_{z}=\frac{\sigma_{0} z}{4 \sqrt{2} \varepsilon s} \cdot e^{\frac{z^{2}}{2 s^{2}}} \int_{\frac{z^{2}}{2 s^{2}}}^{\frac{\left(R^{2}+z^{2}\right)}{2 s^{2}}} w^{-\frac{3}{2}} e^{-w} d w\left[\text { where } w=t+\frac{z^{2}}{2 s^{2}}\right]
\end{aligned}
$$

We have for two functions $u$ and $v$

30 
$d(u v)=u d v+v d u$

$\therefore u v=\int u d v+\int v d u$

$\therefore \int u d v=u v-\int v d u$

Let $u=e^{-w}$ and $d v=w^{-\frac{3}{2}} d w$

Then, we have

$d u=-e^{-w} d w$, and

$v=\int w^{-\frac{3}{2}} d w=\frac{w^{-\frac{1}{2}}}{-\frac{1}{2}}=-2 w^{-\frac{1}{2}}$

Then, we have

$$
\begin{aligned}
& \text { or, } E_{z}=\frac{\sigma_{0} z}{4 \sqrt{2} \varepsilon s} \cdot e^{\frac{z^{2}}{2 s^{2}}} \cdot\left[-2 w^{-\frac{1}{2}} e^{-w} \frac{\left(R^{2}+z^{2}\right)}{2 s^{2}}\right.\left.-\int_{\frac{z^{2}}{2 s^{2}}}^{\frac{\left(R^{2}+z^{2}\right)}{2 s^{2}}} 2 w^{-\frac{1}{2}} e^{-w} d w\right] \\
& \text { or } E_{z}=\frac{\sigma_{0} z}{4 \sqrt{2} \varepsilon s} \cdot e^{\frac{z^{2}}{2 s^{2}}}\left[2\left(\frac{z^{2}}{2 s^{2}}\right)^{-\frac{1}{2}} \cdot e^{-\frac{z^{2}}{2 s^{2}}}-2\left\{\frac{\left(R^{2}+z^{2}\right)}{2 s^{2}}\right\}^{-\frac{1}{2}} \cdot e^{-\frac{\left(R^{2}+z^{2}\right)}{2 s^{2}}}\right. \\
&\left.-2\left\{\Gamma\left(\frac{1}{2}, \frac{z^{2}}{2 s^{2}}\right)-\Gamma\left(\frac{1}{2}, \frac{\left(R^{2}+z^{2}\right)}{2 s^{2}}\right)\right\}\right]
\end{aligned}
$$

or, $E_{z}=\frac{\sigma_{0} z}{2 \sqrt{2} \varepsilon s} \cdot e^{\frac{z^{2}}{2 s^{2}}}\left[\frac{\sqrt{2} s}{|z|} \cdot e^{-\frac{z^{2}}{2 s^{2}}}-\frac{\sqrt{2} s}{\sqrt{R^{2}+z^{2}}} \cdot e^{-\frac{\left(R^{2}+z^{2}\right)}{2 s^{2}}}\right.$

$$
\left.-\left\{\Gamma\left(\frac{1}{2}, \frac{z^{2}}{2 s^{2}}\right)-\Gamma\left(\frac{1}{2}, \frac{\left(R^{2}+z^{2}\right)}{2 s^{2}}\right)\right\}\right]
$$

or, $E_{z}=\frac{\sigma_{0}}{2 \varepsilon}\left[\left(\frac{z}{|z|}-\frac{z}{\sqrt{R^{2}+z^{2}}} \cdot e^{-\frac{R^{2}}{2 s^{2}}}\right)-\frac{z}{\sqrt{2} s} \cdot e^{\frac{z^{2}}{2 s^{2}}} \cdot\left\{\Gamma\left(\frac{1}{2}, \frac{z^{2}}{2 s^{2}}\right)-\Gamma\left(\frac{1}{2}, \frac{\left(R^{2}+z^{2}\right)}{2 s^{2}}\right)\right\}\right]$

From equations (47), (49) and (51), it is evident that the electric field at a point $(0,0, z)$ due to the surface of charge $B$ is given by

$$
E_{B}=\frac{\sigma_{0}}{2 \varepsilon}\left[\left(\frac{z}{|z|}-\frac{z}{\sqrt{R^{2}+z^{2}}} \cdot e^{-\frac{R^{2}}{2 s^{2}}}\right)-\frac{z}{\sqrt{2} s} \cdot e^{\frac{z^{2}}{2 s^{2}}} \cdot\left\{\Gamma\left(\frac{1}{2}, \frac{z^{2}}{2 s^{2}}\right)-\Gamma\left(\frac{1}{2}, \frac{\left(R^{2}+z^{2}\right)}{2 s^{2}}\right)\right\}\right] \ldots
$$

and it is directed along the $z$-axis. 
It may be found by a way of analysis similar as the way shown above that the electric field at a point $(0,0, z)$ due to the surface of charge $\mathrm{B}^{\prime}$ is given by

$$
\begin{aligned}
E_{B^{\prime}}=-\frac{\sigma_{0}}{2 \varepsilon}[ & \left(\frac{(z+2 \delta)}{|(z+2 \delta)|}-\frac{(z+2 \delta)}{\sqrt{b^{2}+(z+2 \delta)^{2}}} \cdot e^{-\frac{b^{2}}{2 s^{2}}}\right) \\
& \left.-\frac{(z+2 \delta)}{\sqrt{2} s} \cdot e^{\frac{(z+2 \delta)^{2}}{2 s^{2}}}\left\{\Gamma\left(\frac{1}{2}, \frac{(z+2 \delta)^{2}}{2 s^{2}}\right)-\Gamma\left(\frac{1}{2}, \frac{b^{2}+(z+2 \delta)^{2}}{2 s^{2}}\right)\right\}\right] \ldots
\end{aligned}
$$

We have for any point inside the insulating disk on the $z$-axis, $z=-|z|$ and $0<|z|<\delta$. Hence, the electric fields at such a point due to the surfaces of charge B and $\mathrm{B}^{\prime}$ are respectively given by

$$
\begin{aligned}
E_{\text {insulator }, B}= & \frac{\sigma_{0}}{2 \varepsilon_{\text {insulator }}}\left[\left(-1+\frac{|z|}{\sqrt{R^{2}+|z|^{2}}} \cdot e^{-\frac{R^{2}}{2 s^{2}}}\right)\right. \\
& \left.+\frac{|z|}{\sqrt{2} s} e^{\frac{|z|^{2}}{2 s^{2}}}\left\{\Gamma\left(\frac{1}{2}, \frac{|z|^{2}}{2 s^{2}}\right)-\Gamma\left(\frac{1}{2}, \frac{\left(R^{2}+|z|^{2}\right)}{2 s^{2}}\right)\right\}\right] \cdots(54), \text { and } \\
E_{\text {insulator }, B^{\prime}}= & -\frac{\sigma_{0}}{2 \varepsilon_{\text {insulator }}}\left[\left(\frac{(2 \delta-|z|)}{|(2 \delta-|z|)|}-\frac{(2 \delta-|z|)}{\sqrt{b^{2}+(2 \delta-|z|)^{2}}} \cdot e^{-\frac{b^{2}}{2 s^{2}}}\right)\right. \\
& -\frac{(2 \delta-|z|)}{\sqrt{2} s} \cdot e^{\frac{(2 \delta-|z|)^{2}}{2 s^{2}}\left\{\Gamma\left(\frac{1}{2}, \frac{(2 \delta-|z|)^{2}}{2 s^{2}}\right)\right.} \\
& \left.\left.-\Gamma\left(\frac{1}{2}, \frac{b^{2}+(2 \delta-|z|)^{2}}{2 s^{2}}\right)\right\}\right] \cdots(55)
\end{aligned}
$$

Equations (54) and (55) may be expressed as shown below.

$$
\begin{aligned}
E_{\text {insulator }, B}= & -\frac{\sigma_{0}}{2 \varepsilon_{\text {insulator }}}\left[\left(1-\frac{|z|}{\sqrt{R^{2}+|z|^{2}}} \cdot e^{-\frac{R^{2}}{2 s^{2}}}\right)\right. \\
& \left.-\frac{|z|}{\sqrt{2} s} e^{\frac{|z|^{2}}{2 s^{2}}}\left\{\Gamma\left(\frac{1}{2}, \frac{|z|^{2}}{2 s^{2}}\right)-\Gamma\left(\frac{1}{2}, \frac{\left(R^{2}+|z|^{2}\right)}{2 s^{2}}\right)\right\}\right] \ldots \\
E_{\text {insulator }, B^{\prime}}= & -\frac{\sigma_{0}}{2 \varepsilon_{\text {insulator }}}\left[\left(1-\frac{(2 \delta-|z|)}{\sqrt{b^{2}+(2 \delta-|z|)^{2}}} \cdot e^{-\frac{b^{2}}{2 s^{2}}}\right)\right. \\
& -\frac{(2 \delta-|z|)}{\sqrt{2} s} \cdot e^{\frac{(2 \delta-|z|)^{2}}{2 s^{2}}\left\{\Gamma\left(\frac{1}{2}, \frac{(2 \delta-|z|)^{2}}{2 s^{2}}\right)\right.} \\
& \left.\left.-\Gamma\left(\frac{1}{2}, \frac{b^{2}+(2 \delta-|z|)^{2}}{2 s^{2}}\right)\right\}\right] \cdots(57)
\end{aligned}
$$

We now proceed to find the electric field at a point $(0,0, z)$ due to the image charges produced in the earthed metal sphere A, the images produced in the earthed metal disk by these image charges, the images produced by these image charges back in sphere A, their images produced again in the earthed metal disk, and so on going on ad infinitum. From table 1 , we have the following. 
Total charge of all the image charges produced at $O$

$$
\begin{aligned}
& =Q_{1}+\frac{a}{2(D+\delta)} Q_{1}+\frac{a^{2}}{\{2(D+\delta)\}^{2}} Q_{1}+\frac{a^{3}}{\{2(D+\delta)\}^{3}} Q_{1}+\frac{a^{4}}{\{2(D+\delta)\}^{4}} Q_{1} \\
& \quad+\cdots \text { to } \infty \text { terms } \\
& =Q_{1}\left[1+\frac{a}{2(D+\delta)}+\frac{a^{2}}{\{2(D+\delta)\}^{2}}+\frac{a^{3}}{\{2(D+\delta)\}^{3}}+\frac{a^{4}}{\{2(D+\delta)\}^{4}}+\cdots \text { to } \infty \text { terms }\right] \\
& =Q_{1} \cdot \frac{1}{\left\{1-\frac{a}{2(D+\delta)}\right\}} \\
& =Q_{1} \cdot \frac{2(D+\delta)}{\{2(D+\delta)-a\}} \\
& =\frac{2(D+\delta)}{\{2(D+\delta)-a\}} Q_{1}
\end{aligned}
$$

$\therefore$ Total charge of all the image charges produced at $O=\frac{2(D+\delta)}{\{2(D+\delta)-a\}} Q_{1} \cdots($

Total charge of all the image charges produced at $O^{\prime}$

$$
\begin{aligned}
& =-Q_{1}-\frac{a}{2(D+\delta)} Q_{1}-\frac{a^{2}}{\{2(D+\delta)\}^{2}} Q_{1}-\frac{a^{3}}{\{2(D+\delta)\}^{3}} Q_{1}-\frac{a^{4}}{\{2(D+\delta)\}^{4}} Q_{1} \\
& \quad-\cdots \text { to } \infty \text { terms } \\
& =-\frac{2(D+\delta)}{\{2(D+\delta)-a\}} Q_{1} \cdots \text { (59) [by evaluating as shown above] }
\end{aligned}
$$

We have

$Q_{1}=q_{i_{1}}+q_{i_{2}}$

Substituting here the expressions for $q_{i_{1}}$ and $q_{i_{2}}$ from equations (26) and (27) respectively, we have

$$
\begin{aligned}
Q_{1}=-\sqrt{2} a s \sigma_{0} \pi e^{\frac{D^{2}}{2 s^{2}}}\left\{\Gamma\left(\frac{1}{2}, \frac{D^{2}}{2 s^{2}}\right)-\Gamma\left(\frac{1}{2}, \frac{R^{2}+D^{2}}{2 s^{2}}\right)\right\} \\
+\sqrt{2} a s \sigma_{0} \pi e^{\frac{(D+2 \delta)^{2}}{2 s^{2}}}\left\{\Gamma\left(\frac{1}{2}, \frac{(D+2 \delta)^{2}}{2 s^{2}}\right)-\Gamma\left(\frac{1}{2}, \frac{b^{2}+(D+2 \delta)^{2}}{2 s^{2}}\right)\right\}
\end{aligned}
$$

Putting this expression for $Q_{1}$ in equations (58) and (59), we get total charge of all the image charges produced at $O$ 


$$
\begin{aligned}
& =\frac{2(D+\delta)}{\{2(D+\delta)-a\}}\left[-\sqrt{2} a s \sigma_{0} \pi e^{\frac{D^{2}}{2 s^{2}}}\left\{\Gamma\left(\frac{1}{2}, \frac{D^{2}}{2 s^{2}}\right)-\Gamma\left(\frac{1}{2}, \frac{R^{2}+D^{2}}{2 s^{2}}\right)\right\}\right. \\
& \left.+\sqrt{2} a s \sigma_{0} \pi e^{\frac{(D+2 \delta)^{2}}{2 s^{2}}}\left\{\Gamma\left(\frac{1}{2}, \frac{(D+2 \delta)^{2}}{2 s^{2}}\right)-\Gamma\left(\frac{1}{2}, \frac{b^{2}+(D+2 \delta)^{2}}{2 s^{2}}\right)\right\}\right] \\
& =-\frac{2(D+\delta)}{\{2(D+\delta)-a\}} \sqrt{2} a s \sigma_{0} \pi\left[e^{\frac{D^{2}}{2 s^{2}}}\left\{\Gamma\left(\frac{1}{2}, \frac{D^{2}}{2 s^{2}}\right)-\Gamma\left(\frac{1}{2}, \frac{R^{2}+D^{2}}{2 s^{2}}\right)\right\}\right. \\
& \left.-e^{\frac{(D+2 \delta)^{2}}{2 s^{2}}}\left\{\Gamma\left(\frac{1}{2}, \frac{(D+2 \delta)^{2}}{2 s^{2}}\right)-\Gamma\left(\frac{1}{2}, \frac{b^{2}+(D+2 \delta)^{2}}{2 s^{2}}\right)\right\}\right] \cdots(60)
\end{aligned}
$$

and

total charge of all the image charges produced at $O^{\prime}$

$$
\begin{aligned}
& =-\frac{2(D+\delta)}{\{2(D+\delta)-a\}}\left[-\sqrt{2} a s \sigma_{0} \pi e^{\frac{D^{2}}{2 s^{2}}}\left\{\Gamma\left(\frac{1}{2}, \frac{D^{2}}{2 s^{2}}\right)-\Gamma\left(\frac{1}{2}, \frac{R^{2}+D^{2}}{2 s^{2}}\right)\right\}\right. \\
& \left.+\sqrt{2} a s \sigma_{0} \pi e^{\frac{(D+2 \delta)^{2}}{2 s^{2}}}\left\{\Gamma\left(\frac{1}{2}, \frac{(D+2 \delta)^{2}}{2 s^{2}}\right)-\Gamma\left(\frac{1}{2}, \frac{b^{2}+(D+2 \delta)^{2}}{2 s^{2}}\right)\right\}\right] \\
& =\frac{2(D+\delta)}{\{2(D+\delta)-a\}} \sqrt{2} a s \sigma_{0} \pi\left[e^{\frac{D^{2}}{2 s^{2}}}\left\{\Gamma\left(\frac{1}{2}, \frac{D^{2}}{2 s^{2}}\right)-\Gamma\left(\frac{1}{2}, \frac{R^{2}+D^{2}}{2 s^{2}}\right)\right\}\right. \\
& \left.-e^{\frac{(D+2 \delta)^{2}}{2 s^{2}}}\left\{\Gamma\left(\frac{1}{2}, \frac{(D+2 \delta)^{2}}{2 s^{2}}\right)-\Gamma\left(\frac{1}{2}, \frac{b^{2}+(D+2 \delta)^{2}}{2 s^{2}}\right)\right\}\right] \cdots(61)
\end{aligned}
$$

The electric field at point $(0,0, z)$ due to all the image charges produced at $\mathrm{O}$ is given by

$$
\begin{aligned}
& E_{O}=\frac{1}{4 \pi \varepsilon(D-z)^{2}} \cdot \frac{2(D+\delta)}{\{2(D+\delta)-a\}} Q_{1} \cdots(62) \text { [using equation (58)] } \\
& \text { or }, E_{O}=-\frac{1}{4 \pi \varepsilon(D-z)^{2}} \frac{2(D+\delta)}{\{2(D+\delta)-a\}} \sqrt{2} a s \sigma_{0} \pi\left[e^{\frac{D^{2}}{2 s^{2}}}\left\{\Gamma\left(\frac{1}{2}, \frac{D^{2}}{2 s^{2}}\right)-\Gamma\left(\frac{1}{2}, \frac{R^{2}+D^{2}}{2 s^{2}}\right)\right\}\right. \\
& -e^{\frac{(D+2 \delta)^{2}}{2 s^{2}}}\left\{\Gamma\left(\frac{1}{2}, \frac{(D+2 \delta)^{2}}{2 s^{2}}\right)\right. \\
& \left.\left.-\Gamma\left(\frac{1}{2}, \frac{b^{2}+(D+2 \delta)^{2}}{2 s^{2}}\right)\right\}\right] \cdots(63)[\text { using equation }(60)]
\end{aligned}
$$

The electric field at point $(0,0, z)$ due to all the image charges produced at $\mathrm{O}^{\prime}$ is given by $E_{O^{\prime}}=-\frac{1}{4 \pi \varepsilon(D+2 \delta+z)^{2}} \frac{2(D+\delta)}{\{2(D+\delta)-a\}} Q_{1} \cdots(64)[u \operatorname{sing}$ equation (59)] 


$$
\begin{aligned}
& \text { or }, E_{O^{\prime}}=\frac{1}{4 \pi \varepsilon(D+2 \delta+z)^{2}} \frac{2(D+\delta)}{\{2(D+\delta)-a\}} \sqrt{2} a s \sigma_{0} \pi\left[e^{\frac{D^{2}}{2 s^{2}}\left\{\Gamma\left(\frac{1}{2}, \frac{D^{2}}{2 s^{2}}\right)\right.}\right. \\
&-\left.\Gamma\left(\frac{1}{2}, \frac{R^{2}+D^{2}}{2 s^{2}}\right)\right\} \\
&-e^{\frac{(D+2 \delta)^{2}}{2 s^{2}}}\left\{\Gamma\left(\frac{1}{2}, \frac{(D+2 \delta)^{2}}{2 s^{2}}\right)\right. \\
&\left.\left.-\Gamma\left(\frac{1}{2}, \frac{b^{2}+(D+2 \delta)^{2}}{2 s^{2}}\right)\right\}\right] \cdots(65)[\text { using equation (61)] }
\end{aligned}
$$

Therefore, the electric fields at any point inside the insulating disk, for which $z=-|z|$ and $0<|z|<\delta$, due to all the image charges produced at $\mathrm{O}$ and due to all the image charges produced at $\mathrm{O}^{\prime}$ are, respectively, given by

$E_{\text {insulator }, O}=\frac{1}{4 \pi \varepsilon_{\text {insulator }}(D+|z|)^{2}} \cdot \frac{2(D+\delta)}{\{2(D+\delta)-a\}} Q_{1} \cdots(66)$ from equation (62)]

or,$E_{\text {insulator, } O}$

$$
\begin{aligned}
& =-\frac{1}{4 \pi \varepsilon_{\text {insulator }}(D+|z|)^{2}} \frac{2(D+\delta)}{\{2(D+\delta)-a\}} \sqrt{2} a s \sigma_{0} \pi\left[e ^ { \frac { D ^ { 2 } } { 2 s ^ { 2 } } } \left\{\Gamma\left(\frac{1}{2}, \frac{D^{2}}{2 s^{2}}\right)\right.\right. \\
& \left.-\Gamma\left(\frac{1}{2}, \frac{R^{2}+D^{2}}{2 s^{2}}\right)\right\} \\
& -e^{\frac{(D+2 \delta)^{2}}{2 s^{2}}}\left\{\Gamma\left(\frac{1}{2}, \frac{(D+2 \delta)^{2}}{2 s^{2}}\right)\right. \\
& \left.\left.-\Gamma\left(\frac{1}{2}, \frac{b^{2}+(D+2 \delta)^{2}}{2 s^{2}}\right)\right\}\right] \cdots(67) \text { [from equation (63)] }
\end{aligned}
$$

and

$$
E_{\text {insulator }, O^{\prime}}=-\frac{1}{4 \pi \varepsilon_{\text {insulator }}(D+2 \delta-|z|)^{2}} \frac{2(D+\delta)}{\{2(D+\delta)-a\}} Q_{1} \cdots
$$

[from equation (64)]

$$
\begin{aligned}
\text { or }, E_{\text {insulator }, O^{\prime}} & \\
& =\frac{1}{4 \pi \varepsilon_{\text {insulator }}(D+2 \delta-|z|)^{2}} \frac{2(D+\delta)}{\{2(D+\delta)-a\}} \sqrt{2} a s \sigma_{0} \pi\left[e ^ { \frac { D ^ { 2 } } { 2 s ^ { 2 } } } \left\{\Gamma\left(\frac{1}{2}, \frac{D^{2}}{2 s^{2}}\right)\right.\right. \\
& \left.-\Gamma\left(\frac{1}{2}, \frac{R^{2}+D^{2}}{2 s^{2}}\right)\right\} \\
& -e^{\frac{(D+2 \delta)^{2}}{2 s^{2}}}\left\{\Gamma\left(\frac{1}{2}, \frac{(D+2 \delta)^{2}}{2 s^{2}}\right)\right. \\
& \left.\left.-\Gamma\left(\frac{1}{2}, \frac{b^{2}+(D+2 \delta)^{2}}{2 s^{2}}\right)\right\}\right] \cdots(69)[\text { from equation (65)] }
\end{aligned}
$$


The net electric field at any point inside the insulating disk on the $z$-axis - for which $z=-|z|$ and $0<|z|<\delta$ - is given by

$E_{\text {insulator, net }}=E_{\text {insulator }, B}+E_{\text {insulator }, B^{\prime}}+E_{\text {insulator }, O}+E_{\text {insulator }, O^{\prime}} \cdots(70)$

where

$$
\begin{aligned}
& E_{\text {insulator }, B}=-\frac{\sigma_{0}}{2 \varepsilon_{\text {insulator }}}\left[\left(1-\frac{|z|}{\sqrt{R^{2}+|z|^{2}}} \cdot e^{-\frac{R^{2}}{2 s^{2}}}\right)\right. \\
& \left.-\frac{|z|}{\sqrt{2} s} e^{\frac{|z|^{2}}{2 s^{2}}}\left\{\Gamma\left(\frac{1}{2}, \frac{|z|^{2}}{2 s^{2}}\right)-\Gamma\left(\frac{1}{2}, \frac{\left(R^{2}+|z|^{2}\right)}{2 s^{2}}\right)\right\}\right] \ldots \\
& E_{\text {insulator }, B^{\prime}}=-\frac{\sigma_{0}}{2 \varepsilon_{\text {insulator }}}\left[\left(1-\frac{(2 \delta-|z|)}{\sqrt{b^{2}+(2 \delta-|z|)^{2}}} \cdot e^{-\frac{b^{2}}{2 s^{2}}}\right)\right. \\
& -\frac{(2 \delta-|z|)}{\sqrt{2} s} \cdot e^{\frac{\left(2 \delta-\left.|z|\right|^{2}\right.}{2 s^{2}}}\left\{\Gamma\left(\frac{1}{2}, \frac{(2 \delta-|z|)^{2}}{2 s^{2}}\right)\right. \\
& \left.\left.-\Gamma\left(\frac{1}{2}, \frac{b^{2}+(2 \delta-|z|)^{2}}{2 s^{2}}\right)\right\}\right] \\
& E_{\text {insulator }, O}=\frac{1}{4 \pi \varepsilon_{\text {insulator }}(D+|z|)^{2}} \cdot \frac{2(D+\delta)}{\{2(D+\delta)-a\}} Q_{1} \cdots(66) \\
& =-\frac{1}{4 \pi \varepsilon_{\text {insulator }}(D+|z|)^{2}} \frac{2(D+\delta)}{\{2(D+\delta)-a\}} \sqrt{2} a s \sigma_{0} \pi\left[e ^ { \frac { D ^ { 2 } } { 2 s ^ { 2 } } } \left\{\Gamma\left(\frac{1}{2}, \frac{D^{2}}{2 s^{2}}\right)\right.\right. \\
& \left.-\Gamma\left(\frac{1}{2}, \frac{R^{2}+D^{2}}{2 s^{2}}\right)\right\} \\
& \left.-e^{\frac{(D+2 \delta)^{2}}{2 s^{2}}}\left\{\Gamma\left(\frac{1}{2}, \frac{(D+2 \delta)^{2}}{2 s^{2}}\right)-\Gamma\left(\frac{1}{2}, \frac{b^{2}+(D+2 \delta)^{2}}{2 s^{2}}\right)\right\}\right]
\end{aligned}
$$

$$
E_{\text {insulator }, O^{\prime}}=-\frac{1}{4 \pi \varepsilon_{\text {insulator }}(D+2 \delta-|z|)^{2}} \frac{2(D+\delta)}{\{2(D+\delta)-a\}} Q_{1} \cdots(68)
$$

or, $E_{\text {insulator }, O^{\prime}}$

$$
\begin{aligned}
& =\frac{1}{4 \pi \varepsilon_{\text {insulator }}(D+2 \delta-|z|)^{2}} \frac{2(D+\delta)}{\{2(D+\delta)-a\}} \sqrt{2} a s \sigma_{0} \pi\left[e ^ { \frac { D ^ { 2 } } { 2 s ^ { 2 } } } \left\{\Gamma\left(\frac{1}{2}, \frac{D^{2}}{2 s^{2}}\right)\right.\right. \\
& \left.-\Gamma\left(\frac{1}{2}, \frac{R^{2}+D^{2}}{2 s^{2}}\right)\right\} \\
& \left.-e^{\frac{(D+2 \delta)^{2}}{2 s^{2}}}\left\{\Gamma\left(\frac{1}{2}, \frac{(D+2 \delta)^{2}}{2 s^{2}}\right)-\Gamma\left(\frac{1}{2}, \frac{b^{2}+(D+2 \delta)^{2}}{2 s^{2}}\right)\right\}\right] \cdots(69)
\end{aligned}
$$


$\therefore E_{\text {insulator, net }}$

$$
\begin{aligned}
& =-\frac{\sigma_{0}}{2 \varepsilon_{\text {insulator }}}\left[\left(1-\frac{|z|}{\sqrt{R^{2}+|z|^{2}}} \cdot e^{-\frac{R^{2}}{2 s^{2}}}\right)-\frac{|z|}{\sqrt{2} s} e^{\frac{|z|^{2}}{2 s^{2}}}\left\{\Gamma\left(\frac{1}{2}, \frac{|z|^{2}}{2 s^{2}}\right)-\Gamma\left(\frac{1}{2}, \frac{\left(R^{2}+|z|^{2}\right)}{2 s^{2}}\right)\right\}\right] \\
& -\frac{\sigma_{0}}{2 \varepsilon_{\text {insulator }}}\left[\left(1-\frac{(2 \delta-|z|)}{\sqrt{b^{2}+(2 \delta-|z|)^{2}}} \cdot e^{-\frac{b^{2}}{2 s^{2}}}\right)\right. \\
& \left.-\frac{(2 \delta-|z|)}{\sqrt{2} s} \cdot e^{\frac{\left(2 \delta-\left.|z|\right|^{2}\right.}{2 s^{2}}}\left\{\Gamma\left(\frac{1}{2}, \frac{(2 \delta-|z|)^{2}}{2 s^{2}}\right)-\Gamma\left(\frac{1}{2}, \frac{b^{2}+(2 \delta-|z|)^{2}}{2 s^{2}}\right)\right\}\right] \\
& -\frac{1}{4 \pi \varepsilon_{\text {insulator }}(D+|z|)^{2}} \frac{2(D+\delta)}{\{2(D+\delta)-a\}} \sqrt{2} a s \sigma_{0} \pi\left[e^{\frac{D^{2}}{2 s^{2}}}\left\{\Gamma\left(\frac{1}{2}, \frac{D^{2}}{2 s^{2}}\right)-\Gamma\left(\frac{1}{2}, \frac{R^{2}+D^{2}}{2 s^{2}}\right)\right\}\right. \\
& \left.-e^{\frac{(D+2 \delta)^{2}}{2 s^{2}}}\left\{\Gamma\left(\frac{1}{2}, \frac{(D+2 \delta)^{2}}{2 s^{2}}\right)-\Gamma\left(\frac{1}{2}, \frac{b^{2}+(D+2 \delta)^{2}}{2 s^{2}}\right)\right\}\right] \\
& +\frac{1}{4 \pi \varepsilon_{\text {insulator }}(D+2 \delta-|z|)^{2}} \frac{2(D+\delta)}{\{2(D+\delta)-a\}} \sqrt{2} a s \sigma_{0} \pi\left[e ^ { \frac { D ^ { 2 } } { 2 s ^ { 2 } } } \left\{\Gamma\left(\frac{1}{2}, \frac{D^{2}}{2 s^{2}}\right)\right.\right. \\
& \left.-\Gamma\left(\frac{1}{2}, \frac{R^{2}+D^{2}}{2 s^{2}}\right)\right\} \\
& \left.-e^{\frac{(D+2 \delta)^{2}}{2 s^{2}}}\left\{\Gamma\left(\frac{1}{2}, \frac{(D+2 \delta)^{2}}{2 s^{2}}\right)-\Gamma\left(\frac{1}{2}, \frac{b^{2}+(D+2 \delta)^{2}}{2 s^{2}}\right)\right\}\right]
\end{aligned}
$$

\section{Results and Discussions}

We have evaluated the electric field that could be produced at the tip of the valve stem of an actual oxygen regulator using our mathematical model. We present the results here. From measurements made for our sample oxygen regulator, we have obtained the following dimensions for our model:

$a=0.8 \mathrm{~mm}, R=27.75 \mathrm{~mm}, b=17.4 \mathrm{~mm}$ and $\delta=1.8 \mathrm{~mm}$.

We have obtained results for three values of the maximum charge density $\sigma_{0}$ on the diaphragm surface: $0.2 \times 10^{-11} \mathrm{C} / \mathrm{mm}^{2}, 0.5 \times 10^{-11} \mathrm{C} / \mathrm{mm}^{2}$ and $1.5 \times 10^{-11} \mathrm{C} / \mathrm{mm}^{2}$. We have tried with these values of charge density following the work of Heidelberg [16]. We have taken $s=b=17.4 \mathrm{~mm}$ in our calculations. In our calculations, we have used the electric permittivity of oxygen gas at NTP. The relative permittivity of oxygen at NTP is 1.00049 [6]. Multiplying it with the permittivity of free space $\left(8.854 \times 10^{-12} \mathrm{~F} / \mathrm{m}\right)$, we obtain the electric permittivity of oxygen gas at NTP as $8.858 \times 10^{-12} \mathrm{~F} / \mathrm{m}$.

First, we present (in Fig. 5) the results for the electric field produced at $\mathrm{P}$ without taking into account the effect of the earthed metallic backing disk, which we have obtained using equation (14). The graphs have been obtained using MATLAB R2014a software. 


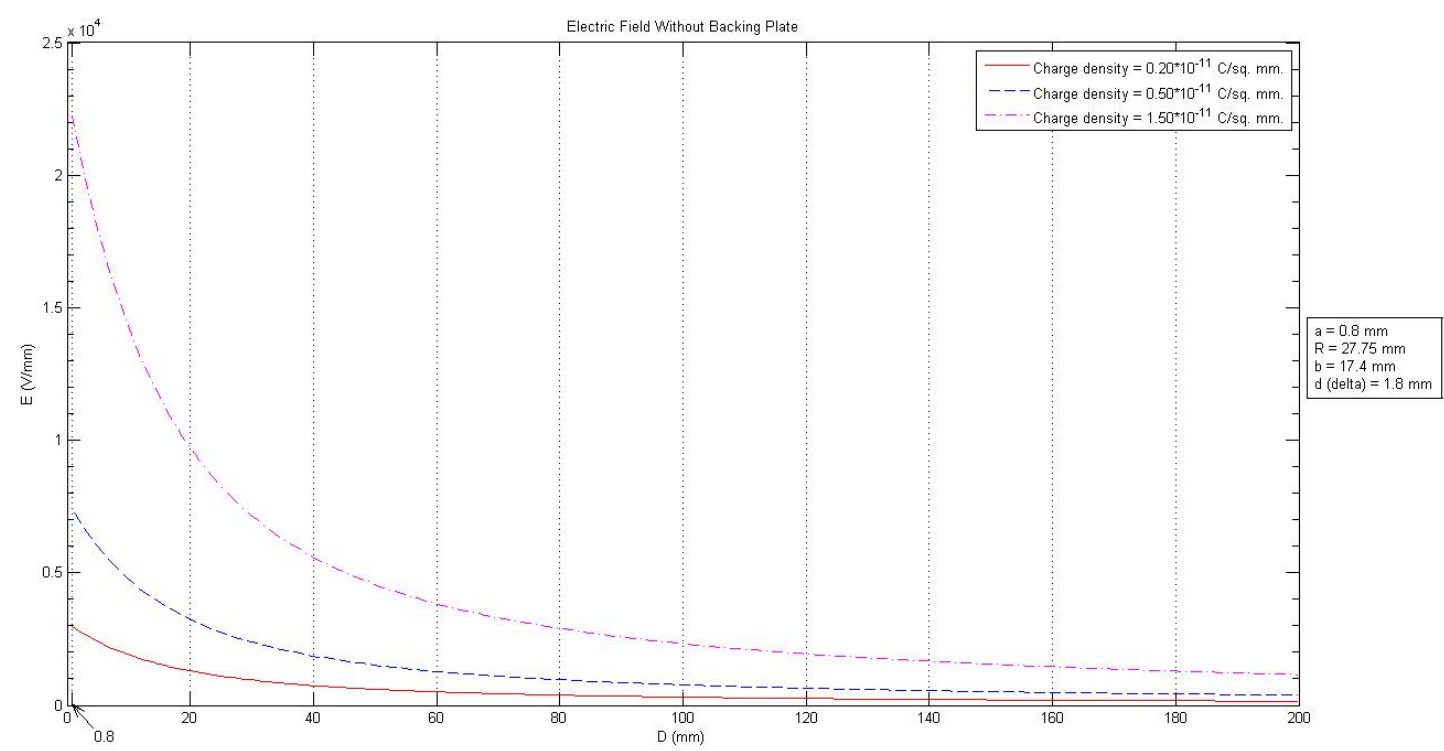

Fig. 5. Electric Field at P Obtained Without Taking into Account the Effect of the Earthed Metal Backing Plate

In Fig. 5 as well as in Fig. 6, the values for the charge density given in the legends are the values of $\sigma_{0}$. As expected, we find that the magnitude of the electric field at $\mathrm{P}$ is maximum when the sphere is almost in contact with the insulating disk, i.e., at $D=0.8 \mathrm{~mm}$, and it decreases as the sphere goes farther and farther away from the disk, i.e., with increasing value of $D$. Also the magnitude of the electric field increases as the charge density on the insulating disk increases.

Now, we present the results obtained for the net electric field at $\mathrm{P}$ discussed in section 3.

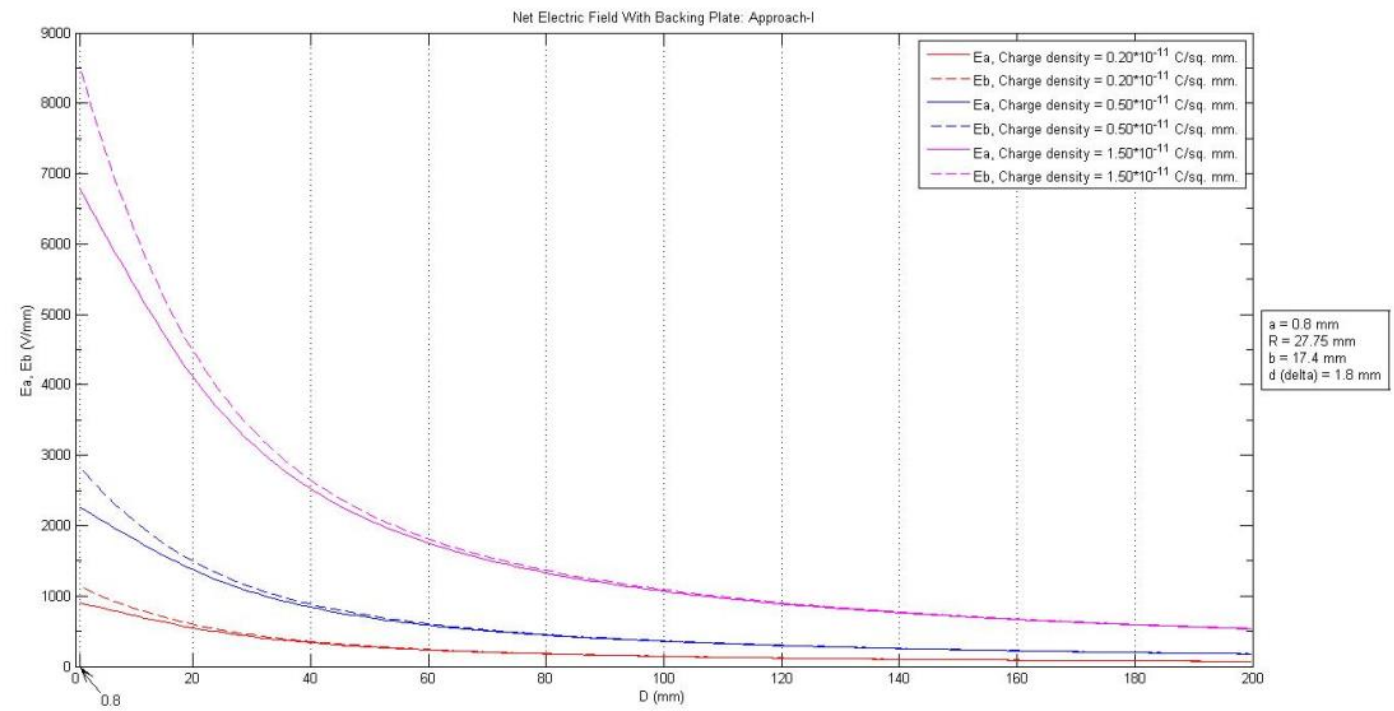

Fig. 6. Results for Net Electric Field at P Obtained Using Our First Approach ('Ea' Representing the Results Obtained With Equation (16) and 'Eb' Representing the Results Obtained With Equation (18))

Fig. 6 depicts the results for the net electric field at P obtained using both equation (16) and equation (18). The graphs have been obtained using MATLAB R2014a. We note here that the 
magnitudes of the net electric field for all the three values of $\sigma_{0}$ are considerably less (by one order of magnitude) than the corresponding values obtained without taking into account the effect of the backing plate. It is, thus, clear that the earthed backing disk has the effect of reducing the magnitude of the electric field produced at $\mathrm{P}$. We also note that the difference between the results obtained using equations (16) and (18) is negligible for large distances of separation between the earthed sphere and the insulating disk. The difference starts becoming visible at about $D=80 \mathrm{~mm}$ for $\sigma_{0}=1.5 \times 10^{-11} \mathrm{C} / \mathrm{mm}^{2}$, at about $D=40 \mathrm{~mm}$ for $\sigma_{0}=0.5 \times$ $10^{-11} \mathrm{C} / \mathrm{mm}^{2}$ and at about $D=20 \mathrm{~mm}$ for $\sigma_{0}=0.2 \times 10^{-11} \mathrm{C} / \mathrm{mm}^{2}$. Also, the absolute difference is greater for greater values of the charge density. The results for the net electric field obtained from equation (18) are greater than those obtained from equation (16) by a maximum (occurring at $D=0.8 \mathrm{~mm}$ ) of $25.4 \%$ for all the three values of $\sigma_{0}$. This difference goes down to $9 \%$ at $D=20 \mathrm{~mm}$ and to less than $5 \%$ at $D=40 \mathrm{~mm}$ for all the three values of $\sigma_{0}$.

Our final expression for the net electric field at $\mathrm{P}$ given by equation (34) (which is by far the most correct expression for this electric field) will produce a curve which lies in between the two curves (one curve obtained using equation (16) and the other curve obtained using equation (18)) shown for each value of $\sigma_{0}$ in fig. 6 . The graphs that have been obtained for the net electric field at $\mathrm{P}$ using this expression for the three values of $\sigma_{0}$ that we have considered here $\left(0.2 \times 10^{-11} \mathrm{C} / \mathrm{mm}^{2}, 0.5 \times 10^{-11} \mathrm{C} / \mathrm{mm}^{2}\right.$ and $\left.1.5 \times 10^{-11} \mathrm{C} / \mathrm{mm}^{2}\right)$ are shown below (in Fig. 7, Fig. 8 and Fig. 9 respectively). These graphs have been obtained using the Wolfram Development Platform (https://develop.open.wolframcloud.com/app/). 


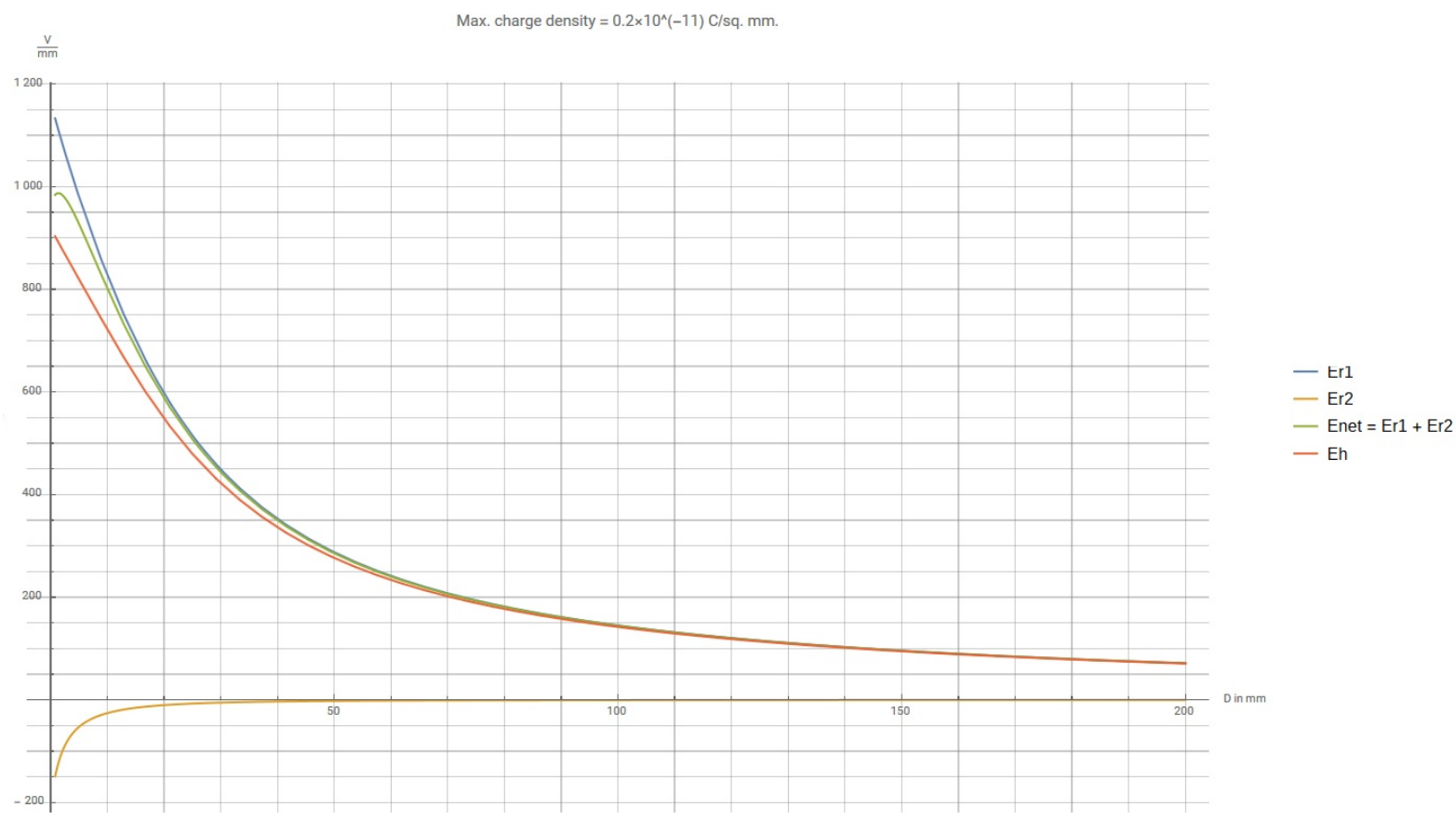

Fig. 7. Plot of the Net Electric Field at P Given By Equation (34) (Denoted Here By Enet), Plots of Its Components, Er1 and Er2, Er1 Being the Component Due to the Charges on the Charged Surface of the Insulating Disk and Their Images Produced Due to Their Reflection on the Earthed Metal Disk and the Images of All these Charges Produced in the Earthed Metal Sphere, and Er2 Being the Component Due to the Reflection of the Image Charges Produced in the Earthed Metal Sphere on the Earthed Metal Disk, and the Reflection of the New Image Charges Produced in the Earthed Metal Disk Due to the Abovementioned Reflection on the Earthed Metal Sphere, and the Reflection of the New Image Charges Produced in the Earthed Metal Sphere Due to the Aforesaid Reflection on the Earthed Metal Disk, and Such Reflections Going On on the Two Metal Surfaces Ad Infinitum, and a Plot of the Net Electric Field at P Given By Equation (16) Obtained Going By the Suggestion of Heidelberg (Denoted Here by Eh) for $\sigma_{0}=0.2 \times 10^{-11} \mathrm{C} / \mathrm{mm}^{2}$ 




Fig. 8. Plot of the Net Electric Field at P Given By Equation (34) (Denoted Here By Enet), Plots of Its Components, Er1 and Er2, Er1 Being the Component Due to the Charges on the Charged Surface of the Insulating Disk and Their Images Produced Due to Their Reflection on the Earthed Metal Disk and the Images of All these Charges Produced in the Earthed Metal Sphere, and Er2 Being the Component Due to the Reflection of the Image Charges Produced in the Earthed Metal Sphere on the Earthed Metal Disk, and the Reflection of the New Image Charges Produced in the Earthed Metal Disk Due to the Abovementioned Reflection on the Earthed Metal Sphere, and the Reflection of the New Image Charges Produced in the Earthed Metal Sphere Due to the Aforesaid Reflection on the Earthed Metal Disk, and Such Reflections Going On on the Two Metal Surfaces Ad Infinitum, and a Plot of the Net Electric Field at P Given By Equation (16) Obtained Going By the Suggestion of Heidelberg (Denoted Here by Eh) for $\sigma_{0}=0.5 \times 10^{-11} \mathrm{C} / \mathrm{mm}^{2}$ 


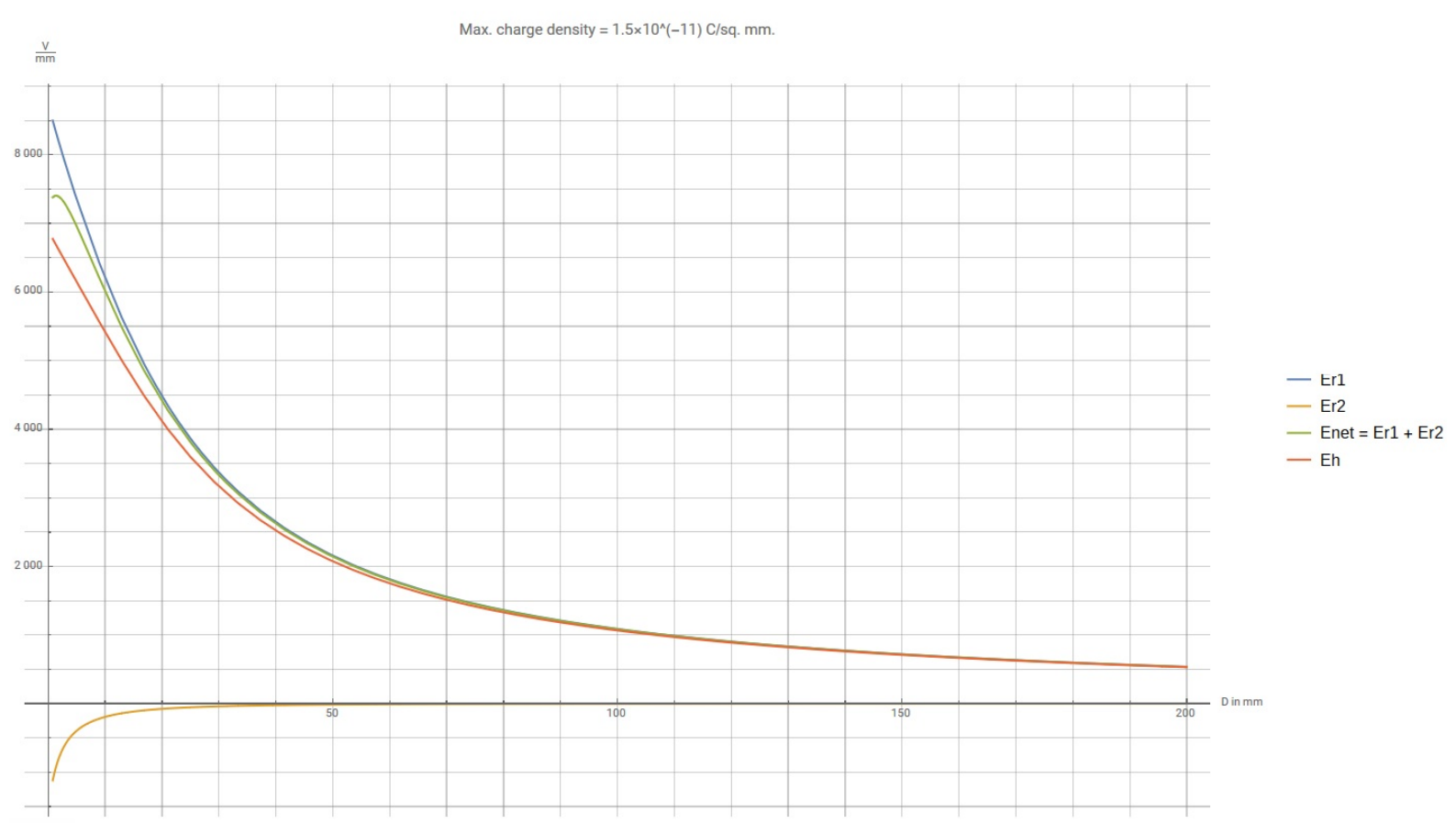

Fig. 9. Plot of the Net Electric Field at P Given By Equation (34) (Denoted Here By Enet), Plots of Its Components, Er1 and Er2, Er1 Being the Component Due to the Charges on the Charged Surface of the Insulating Disk and Their Images Produced Due to Their Reflection on the Earthed Metal Disk and the Images of All these Charges Produced in the Earthed Metal Sphere, and Er2 Being the Component Due to the Reflection of the Image Charges Produced in the Earthed Metal Sphere on the Earthed Metal Disk, and the Reflection of the New Image Charges Produced in the Earthed Metal Disk Due to the Abovementioned Reflection on the Earthed Metal Sphere, and the Reflection of the New Image Charges Produced in the Earthed Metal Sphere Due to the Aforesaid Reflection on the Earthed Metal Disk, and Such Reflections Going On on the Two Metal Surfaces Ad Infinitum, and a Plot of the Net Electric Field at P Given By Equation (16) Obtained Going By the Suggestion of Heidelberg (Denoted Here by Eh) for $\sigma_{0}=1.5 \times 10^{-11} \mathrm{C} / \mathrm{mm}^{2}$

Now, we shall find out the breakdown strength of an oxygen-enriched atmosphere considering our system shown in fig. 2 using Bower's criterion [16]. The criterion is as follows.

$$
e^{\frac{v}{\lambda E_{\text {breakdown }}}}=\frac{a E_{\text {breakdown }}}{2 v}-\frac{a \lambda E_{\text {breakdown }}^{2}}{4 v^{2}} \ldots(43),
$$

where

$v$ is the ionization potential of the surrounding gas (we take $12.5 \mathrm{~V}$ for its value for the case of oxygen),

$\lambda$ is the mean free path of an electron (we take $3.7 \times 10^{-7} \mathrm{~m}$. for its value), and $a$ is the radius of the earthed metal sphere (which is $0.8 \mathrm{~mm}$. for our case).

Putting these values in equation (43), we get

$$
e^{\frac{3.38 \times 10^{7}}{E_{\text {breakdown }}}}=3.2 \times 10^{-5} E_{\text {breakdown }}-4.7 \times 10^{-13} E_{\text {breakdown }} \ldots
$$


Plotting the expressions on the left-hand side and the right-hand side of this equation against $E_{\text {breakdown }}$ (using Wolfram Alpha Pro - http://www.wolframalpha.com), we get the following graphs (shown in Fig. 10).



Fig. 10. Graphically Solving Bower's Equation (Equation (44)) Using Wolfram Alpha Pro (http://www.wolframalpha.com)

We find in Fig. 10 that the graphs intersect at about $E_{\text {breakdown }}=0.65 \times 10^{7} \mathrm{~V} / \mathrm{m}$.

Thus, the solution of equation (44) is

$$
\begin{aligned}
E_{\text {breakdown }} & =0.65 \times 10^{7} \mathrm{~V} / \mathrm{m} . \\
& =65 \mathrm{KV} / \mathrm{cm} . \\
& =6.5 \mathrm{KV} / \mathrm{mm} .
\end{aligned}
$$

It may be observed from Fig. 9 that considering the value of the breakdown electric field of the oxygen gas in the regulator to be $6.5 \mathrm{kV} / \mathrm{mm}$., an electrostatic discharge would ensue when the earthed metal sphere comes at a distance of $(7.5-0.8)$ mm., i.e., $6.7 \mathrm{~mm}$. from charged surface of the insulating disk if the maximum charge density $\left(\sigma_{0}\right)$ on the disk is $1.5 \times 10^{-11} \mathrm{C} / \mathrm{mm}^{2}$. It thus appears that the occurrence of an electrostatic discharge inside an oxygen regulator is a possibility.

Now, we can also calculate the minimum value of $\sigma_{0}$ which is required for a discharge to occur at point $\mathrm{P}$ using equation (42) and equation (41). For the dimensions we have used for our calculations, we have

$f_{2}(\delta, a, b, R, s)=8.675[$ using equation $(41)]$. 
Then, using $\varepsilon=8.858 \times 10^{-12} \mathrm{~F} / \mathrm{m}=8.858 \times 10^{-15} \mathrm{~F} / \mathrm{mm}$ and $E_{\text {breakdown }}=6.5 \mathrm{KV} / \mathrm{mm}$, we get

$$
\begin{aligned}
& \sigma_{0}=2 \varepsilon E_{\text {breakdown }}\left[f_{2}(\delta, a, R, b, s)\right]^{-1} \text { [equation (42)] } \\
& \text { or, } \sigma_{0}=1.33 \times 10^{-11} \mathrm{C} / \mathrm{mm}^{2}
\end{aligned}
$$

This is the minimum value of $\sigma_{0}$ which is required for a discharge to occur at point $\mathrm{P}$, the point of the earthed sphere nearest to the charged surface of the insulator and which lies on the perpendicular to the insulating disk at its centre. We may also call this charge density on the insulating surface the 'critical charge density'.

The minimum value of $\sigma_{0}$ which is required for a discharge to occur at point $\mathrm{P}$ when the centre of the earthed metal sphere is at a distance $D$ from the charged surface of the insulating disk has been calculated for various values of $D$ for the dimensions we have used here using equation (42 A). The graph thus obtained is shown in the fig. 11 given below.

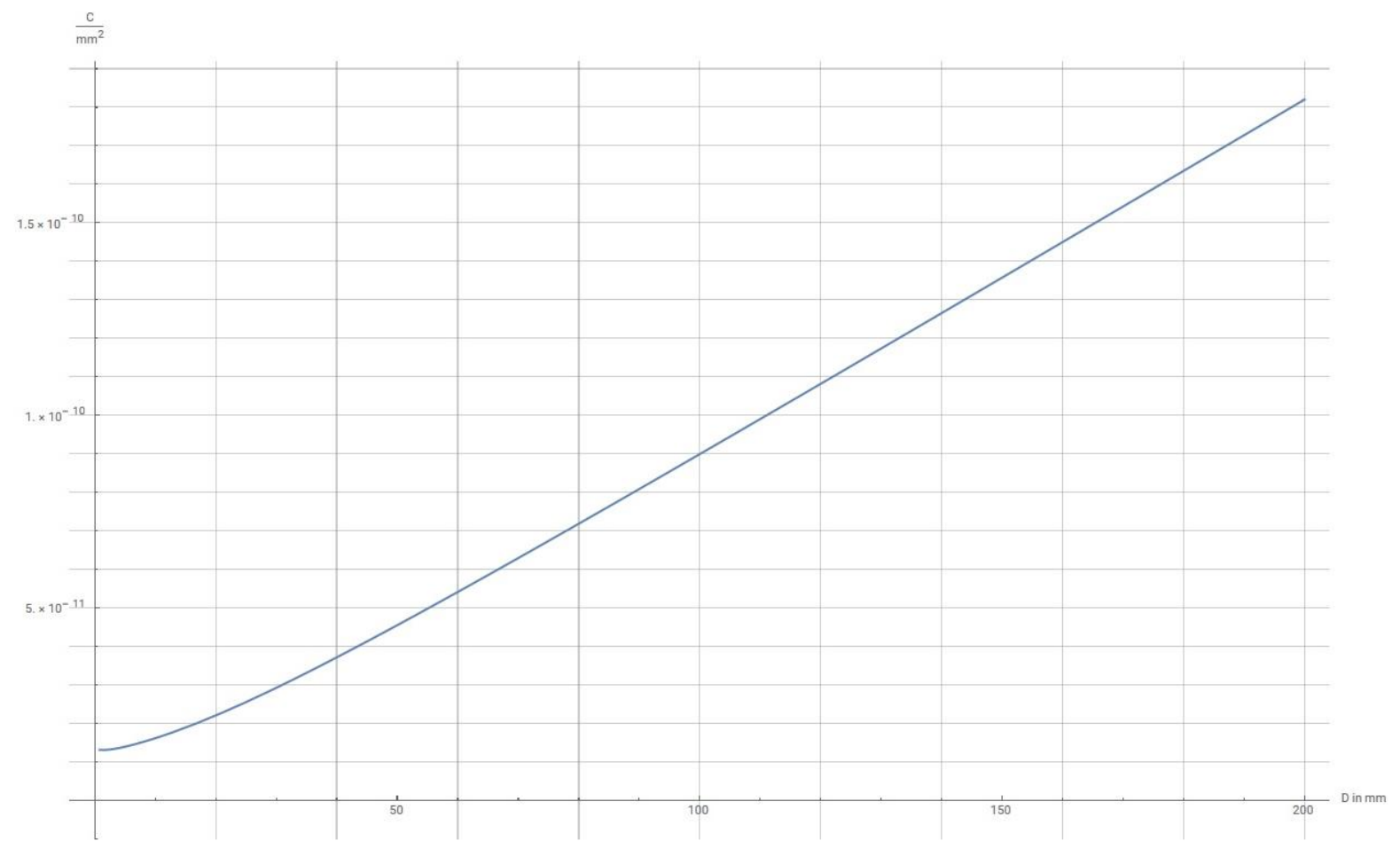

Fig. 11. The Minimum Value of $\sigma_{0}$ Which is Required for a Discharge to Occur at Point $\mathrm{P}$ When the Centre of the Earthed Metal Sphere Is at a Distance $D$ from the Charged Surface of the Insulating Disk

From the above results and discussions, we see that there is a possibility that an electrostatic discharge could occur inside an oxygen regulator. Now, considering the fact that the atmosphere inside an oxygen regulator is oxygen enriched for the most part, it also seems likely that such a discharge could also be responsible for fire hazards.

The electric field inside the insulating disk has been calculated using equation (71) for the dimensions used here for the three values of $\sigma_{0}$ that we have considered here $\left(0.2 \times 10^{-11}\right.$ $\mathrm{C} / \mathrm{mm}^{2}, 0.5 \times 10^{-11} \mathrm{C} / \mathrm{mm}^{2}$ and $1.5 \times 10^{-11} \mathrm{C} / \mathrm{mm}^{2}$ ) and also for $\sigma_{0}=1.33 \times 10^{-11} \mathrm{C} / \mathrm{mm}^{2}$ 
which has been found to be the critical charge density for various values of $\mathrm{D}$ and $z$. In doing these calculations, the relative permittivity of neoprene, which is 6.7 [17], has been used as the diaphragms of many oxygen regulators are made of neoprene [18]. Graphs for this electric field have been plotted using the Wolfram Development Platform (https://develop.open.wolframcloud.com/app/). These graphs are shown below.



Fig. 12. Plots of the Net Electric Field at a point $z$ on the $z$-axis Inside the Insulating Disk (Denoted Here By Enet) Versus $z$ and of Its Components, Er1 and Er2, Er1 Being the Component Due to the Charges on the Charged Surface of the Insulating Disk, i.e., the Surface of Charge B and Their Images Produced Due to Their Reflection on the Earthed Metal Disk, i.e., the Surface of Charge B', and Er2 Being the Component Due to the Image Charges Produced in the Earthed Metal Sphere Due to Reflection of the Charges on the Surfaces of Charge B and B', and the Reflection of the Image Charges Produced in the Earthed Metal Sphere on the Earthed Metal Disk, and the Reflection of the New Image Charges Produced in the Earthed Metal Disk Due to the Abovementioned Reflection on the Earthed Metal Sphere, and the Reflection of the New Image Charges Produced in the Earthed Metal Sphere Due to the Aforesaid Reflection on the Earthed Metal Disk, and Such Reflections Going On on the Two Metal Surfaces Ad Infinitum for $\sigma_{0}=0.2 \times 10^{-11} \mathrm{C} / \mathrm{mm}^{2}$ 


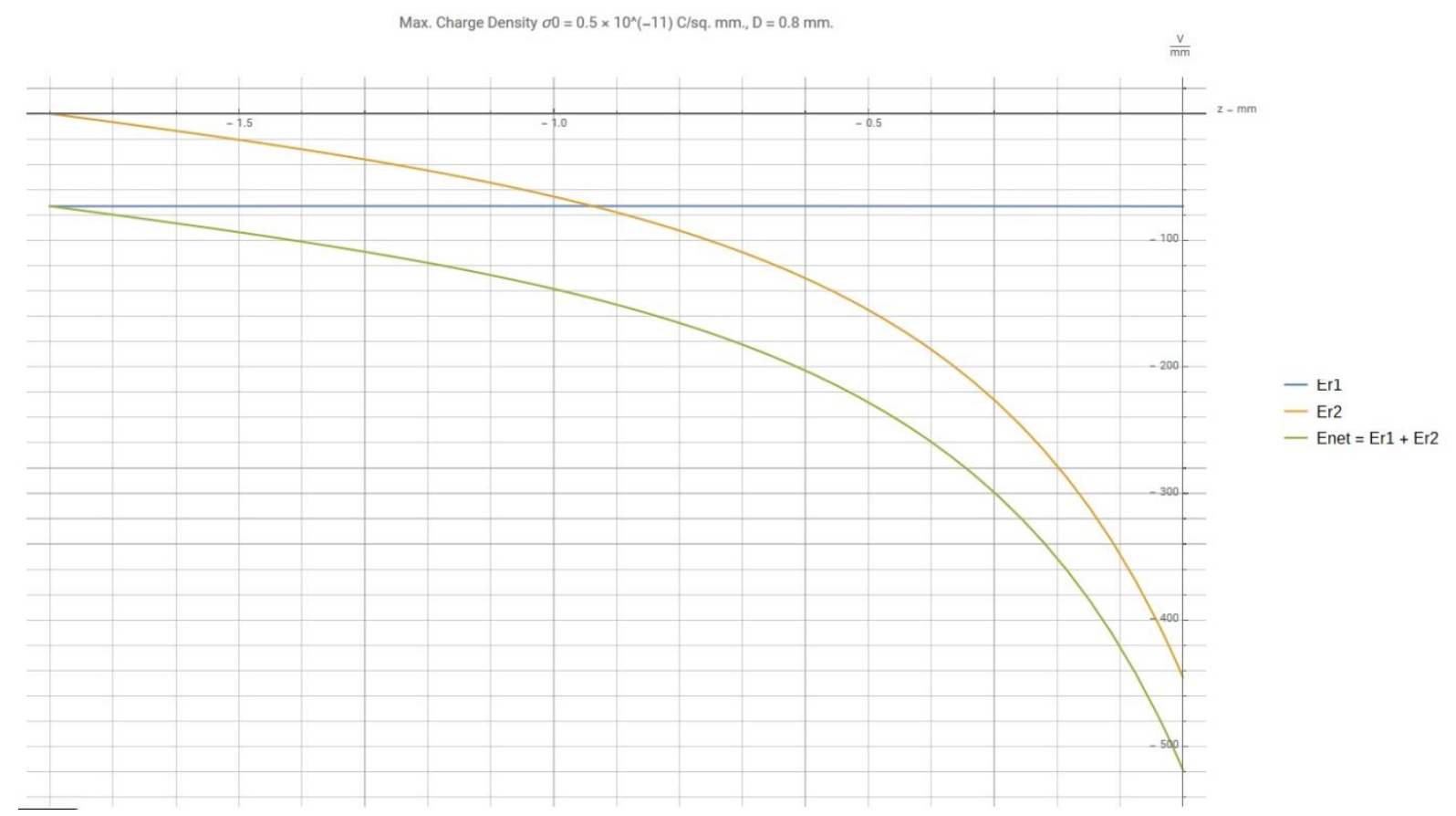

Fig. 13. Plots of the Net Electric Field at a point $z$ on the $z$-axis Inside the Insulating Disk (Denoted Here By Enet) Versus $z$ and of Its Components, Er1 and Er2, Er1 Being the Component Due to the Charges on the Charged Surface of the Insulating Disk, i.e., the Surface of Charge B and Their Images Produced Due to Their Reflection on the Earthed Metal Disk, i.e., the Surface of Charge B', and Er2 Being the Component Due to the Image Charges Produced in the Earthed Metal Sphere Due to Reflection of the Charges on the Surfaces of Charge B and B', and the Reflection of the Image Charges Produced in the Earthed Metal Sphere on the Earthed Metal Disk, and the Reflection of the New Image Charges Produced in the Earthed Metal Disk Due to the Abovementioned Reflection on the Earthed Metal Sphere, and the Reflection of the New Image Charges Produced in the Earthed Metal Sphere Due to the Aforesaid Reflection on the Earthed Metal Disk, and Such Reflections Going On on the Two Metal Surfaces Ad Infinitum for $\sigma_{0}=0.5 \times 10^{-11} \mathrm{C} / \mathrm{mm}^{2}$ 




Fig. 14. Plots of the Net Electric Field at a point $z$ on the $z$-axis Inside the Insulating Disk (Denoted Here By Enet) Versus $z$ and of Its Components, Er1 and Er2, Er1 Being the Component Due to the Charges on the Charged Surface of the Insulating Disk, i.e., the Surface of Charge B and Their Images Produced Due to Their Reflection on the Earthed Metal Disk, i.e., the Surface of Charge B', and Er2 Being the Component Due to the Image Charges Produced in the Earthed Metal Sphere Due to Reflection of the Charges on the Surfaces of Charge B and B', and the Reflection of the Image Charges Produced in the Earthed Metal Sphere on the Earthed Metal Disk, and the Reflection of the New Image Charges Produced in the Earthed Metal Disk Due to the Abovementioned Reflection on the Earthed Metal Sphere, and the Reflection of the New Image Charges Produced in the Earthed Metal Sphere Due to the Aforesaid Reflection on the Earthed Metal Disk, and Such Reflections Going On on the Two Metal Surfaces Ad Infinitum for $\sigma_{0}=1.5 \times 10^{-11} \mathrm{C} / \mathrm{mm}^{2}$ 


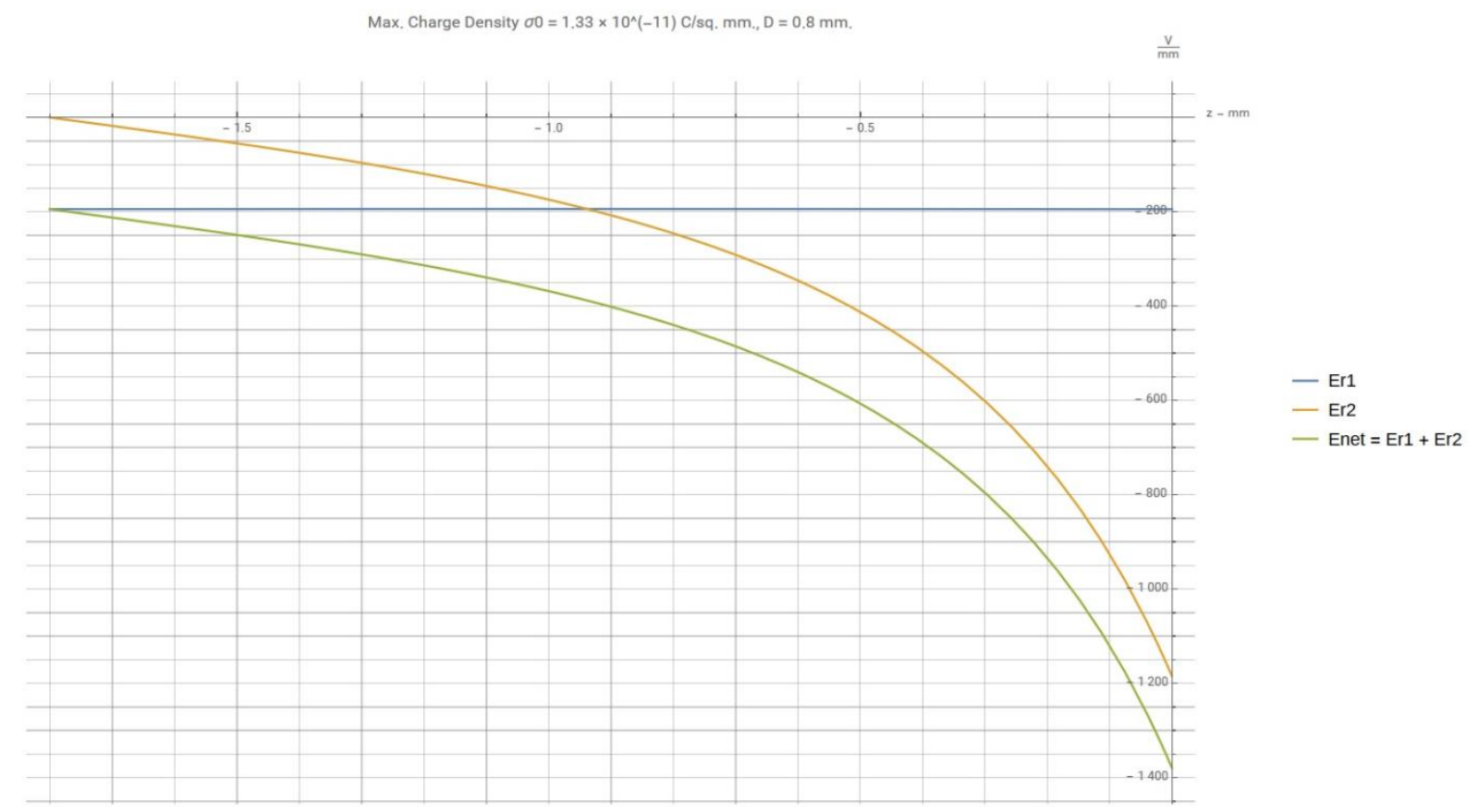

Fig. 15. Plots of the Net Electric Field at a point $z$ on the $z$-axis Inside the Insulating Disk (Denoted Here By Enet) Versus $z$ and of Its Components, Er1 and Er2, Er1 Being the Component Due to the Charges on the Charged Surface of the Insulating Disk, i.e., the Surface of Charge B and Their Images Produced Due to Their Reflection on the Earthed Metal Disk, i.e., the Surface of Charge B', and Er2 Being the Component Due to the Image Charges Produced in the Earthed Metal Sphere Due to Reflection of the Charges on the Surfaces of Charge B and B', and the Reflection of the Image Charges Produced in the Earthed Metal Sphere on the Earthed Metal Disk, and the Reflection of the New Image Charges Produced in the Earthed Metal Disk Due to the Abovementioned Reflection on the Earthed Metal Sphere, and the Reflection of the New Image Charges Produced in the Earthed Metal Sphere Due to the Aforesaid Reflection on the Earthed Metal Disk, and Such Reflections Going On on the Two Metal Surfaces Ad Infinitum for $\sigma_{0}=1.33 \times 10^{-11} \mathrm{C} / \mathrm{mm}^{2}$

The breakdown electric field strength of neoprene lies between $15.7 \mathrm{MV} / \mathrm{m}$. to $26.7 \mathrm{MV} / \mathrm{m}$. [19], i.e., between $15.7 \mathrm{KV} / \mathrm{mm}$. to $26.7 \mathrm{KV} / \mathrm{mm}$.. We can see from the graphs above that the electric field inside the neoprene diaphragm does not reach such values for values of $\sigma_{0}$ upto $1.5 \times 10^{-11} \mathrm{C} / \mathrm{mm}^{2}$. The electric field inside the neoprene diaphragm is expected to reach the breakdown electric field strength of neoprene at much higher values of $\sigma_{0}$.

\section{Conclusion}

Thus, in trying to investigate the electric field which is likely to be produced at the tip of the valve stem inside a typical oxygen regulator due to the charge that might be produced on the diaphragm, we have first modelled the system as a very small earthed metal sphere in front of a charged insulating disk partially backed by an earthed metal disk. We have qualitatively argued that the density of charge that could be produced on the diaphragm might suitably be represented by the Gaussian function. We have then derived an analytical expression (equation (34)) for the net electric field produced at the point on the earthed metal sphere nearest to the charged surface of the insulating disk. Next, we have calculated the magnitude of the electric field produced at this tip of the earthed sphere in our model using 
measurements obtained from a sample oxygen regulator for the dimensions of the various components of our model. We have also found out an expression for the critical charge density on the surface of the insulating disk (equation (42)), and have also found out a value for this critical charge density for the sample oxygen regulator we have considered, which is $1.33 \times 10^{-11} \mathrm{C} / \mathrm{mm}^{2}$. We have also found out an expression for the electric field inside the insulating disk in our system (equation (71)), and have calculated values of it for the sample oxygen regulator we have considered here. It appears from our analyses that there is a possibility that an electrostatic discharge could occur inside an oxygen regulator, and due to the presence of an enriched-oxygen atmosphere, such a discharge might also lead to a fire incident.

\section{References}

1. "Oxygen Regulator Fire Severely Burns One Fire Fighter - Florida", A Summary of a NIOSH Fire Fighter Fatality Investigation, NIOSH, February 5, 1999.

2. "Emergency Medical Technician Receives Serious Burns from an Oxygen Regulator Flash Fire - South Carolina", A Summary of a NIOSH Fire Fighter Injury Investigation, NIOSH, September 13, 1999.

3. U.S. Fire Administration/Technical Report Series, "Special Report: Fires Involving Medical Oxygen Equipment", USFA-TR-107, FEMA, March 1999.

4. D. W. G. Dicker and R. K. Wharton, "A Review of Incidents Involving the Use of High-pressure Oxygen from 1982 to 1985 in Great Britain", Flammability and Sensitivity of Materials in Oxygenenriched Atmospheres: Third Volume, ASTM STP 986, D. W. Schroll, Ed., American Society for Testing and Materials, Philadelphia, 1988, pp. 318-327.

5. F. E. Kelly, R. Hardy, E. A. Hall, J. McDonald, M. Turner, J. Rivers, H. Jones, J. P. Nolan, T. M. Cook and P. Henrys, "Fire on an Intensive Care Unit Caused by an Oxygen Cylinder", Anaesthesia 2012, pp. $1-3$.

6. H. D. Beeson, S. R. Smith and W. F. Stewart, Ed., Safe Use of Oxygen and Oxygen Systems: Handbook for Design, Operation, and Maintenance, Second Edition, ASTM Stock Number: MNL362nd, ASTM International, 2007.

7. T. D. Gallus and J. M. Stoltzfus, "Flow Friction Fire History and Research", Journal of ASTM International, Vol. 3, No. 9, 2006, pp. 151-162.

8. J. D. Hooser, M. Wei, B. E. Newton and G. J. A. Chiffoleau, "An Approach to Understanding Flow Friction Ignition: A Computational Fluid Dynamics (CFD) Study on Temperature Development of High-Pressure Oxygen Flow Inside Micron-Scale Seal Cracks", Journal of ASTM International, Vol. 6, No. 10, 2009.

9. A. Oza, S. Ghosh and K. Chowdhury, "Tribocharging of Particle Contaminants Evaluated as an Ignition Source in Oxygen-Enriched Environments", Journal of ASTM International, Vol. 6, No. 8, 2009, pp. 389-428.

10. O. A. Kirit, "Evaluation of Electrostatic Discharge Due to Tribocharging of Contaminant Particles as an Ignition Mechanism in Oxygen Systems", Ph. D. Thesis, Cryogenic Engineering Centre, Indian Institute of Technology Kharagpur, April 2010. 
11. C. I. Calle, J. G. Mantovani, C. R. Buhler, M.D. Hogue, A. W. Nowicki and E. E. Groop, "Electrostatic Charging of Polymers by Particle Impact at Low Pressures", The Fourth International Conference on Applied Electrostatics, 2001 Dalian.

12. E. Heidelberg, "Generation of Igniting Brush Discharges by Charged Layers on Earthed Conductors", Static Electrification, Proceedings of the Conference Organized by The Institute of Physics and the Physical Society, London, May 1967, pp. $147-155$.

13. A. Oza, S. Ghosh and K. Chowdhury, "Modeling of Dispersion and Settling of Micron-SizedDust Particles in Pressure Regulator Used in Oxygen-Enriched Environment", Journal of ASTM International, Vol. 8, No. 4, 2011, pp. 1 - 19.

14. R. P. Feynman, R. Leighton, M. Sands, "The Feynman Lectures on Physics", Vol. 2, online edition, California Institute of Technology. (http://www.feynmanlectures.caltech.edu)

15. Sir J. Jeans, "The Mathematical Theory of Electricity and Magnetism", Cambridge, 1911.

16. E. Heidelberg, "Test and Judgement of Ignition Hazards Caused by Electrostatic Spray Guns and Charged Surfaces Without the Use of Explosive Mixtures", Journal of Electrostatics, Vol. 21, 1988, pp. $1-17$.

17. Hugh D. Young, Roger A. Freedman and A. Lewis Ford, "Sears and Zemansky's University Physics", Addison-Wesley, 2012.

18. https://www.thefabricator.com/article/oxyfuelcutting/what-s-there-to-know-about-regulators-

19. https://en.wikipedia.org/wiki/Dielectric_strength 